\title{
THE VISHIK-LYUSTERNIK METHOD IN ELLIPTIC PROBLEMS WITH A SMALL PARAMETER
}

\author{
L. R. VOLEVICH
}

To B. P. Paneyakh for his 70th birthday

\begin{abstract}
We consider boundary value problems where the operator defined in a domain and the boundary operators depend on a small parameter. Elliptic and properly elliptic problems with a small parameter are defined. It is proved that small parameter ellipticity is a necessary and sufficient condition for the existence of a priori estimates that are uniform with respect to the parameter. The proof of uniform estimates is based on the construction of the exponential boundary layer introduced in the classical paper by Vishik and Lyusternik.
\end{abstract}

\section{INTRODUCTION}

The problem we study in this paper can be formulated as follows. On a manifold $M$ with a smooth boundary $\partial M$ we consider the following problem for an elliptic operator of order $2 m$ :

$$
\begin{gathered}
A(x, D, \varepsilon) u(x)=f(x), \quad x \in M, \\
B_{j}\left(x^{\prime}, D, \varepsilon\right) u\left(x^{\prime}\right)=g_{j}\left(x^{\prime}\right), \quad x^{\prime} \in \partial M, \quad j=1, \ldots, m,
\end{gathered}
$$

where the operators in (0.1) and (0.2) depend polynomially on a small parameter $\varepsilon$ :

$$
\begin{aligned}
A(x, D, \varepsilon) & :=\varepsilon^{2 m-2 \mu} A_{2 m}(x, D)+\varepsilon^{2 m-2 \mu-1} A_{2 m-1}(x, D)+\cdots+A_{2 \mu}(x, D), \\
B_{j}\left(x^{\prime}, D, \varepsilon\right) & :=\varepsilon^{b_{j}-\beta_{j}} B_{j, b_{j}}\left(x^{\prime}, D\right)+\varepsilon^{b_{j}-\beta_{j}-1} B_{j, b_{j}-1}\left(x^{\prime}, D\right)+\cdots+B_{j, \beta_{j}}\left(x^{\prime}, D\right) .
\end{aligned}
$$

Here by $A_{2 m-j}, j=0, \ldots, 2 m-2 \mu(\mu<m)$ and $B_{j, b_{j}-k}, k=0, \ldots, b_{j}-\beta_{j}\left(\beta_{j}<b_{j}\right)$ we denote differential operators of orders $2 m-j$ and $b_{j}-k$, respectively, with leading homogeneous terms $A_{2 m-j}^{0}$ and $B_{j, b_{j}-k}^{0}$.

Replacing in (0.3) $A_{2 m-j}$ with $A_{2 m-j}^{0}$ we obtain the leading term $A^{0}(x, D, \varepsilon)$ of the operator $A(x, D, \varepsilon)$. Similarly, replacing in (0.4) $B_{j, b_{j}-k}$ with $B_{j, b_{j}-k}^{0}$ we obtain the leading term $B_{j}^{0}\left(x^{\prime}, D, \varepsilon\right)$ of the boundary operator (0.4).

0.1. Although specific problems of the type (0.1), (0.2) with operators of the form (0.3), (0.4) did appear quite some time ago in various areas of mathematical physics (especially in elasticity) and were well known for quite some time, a comprehensive theory of such operators had started up with a remarkable paper (essentially a monograph) by M. I. Vishik and L. A. Lyusternik 11. In this paper the main condition on the dependence of the operator (0.1) on a small parameter was formulated and the asymptotics as $\varepsilon \rightarrow 0$ of the solution of the Dirichlet problem was constructed. In the proof of this asymptotics the condition of strong ellipticity of the operator (0.3) was used.

The main feature of the problem (0.1), (0.2) is that at $\varepsilon=0$ we obtain an elliptic equation of order $2 \mu<2 m$, which requires only $\mu$ boundary conditions. Therefore

2000 Mathematics Subject Classification. Primary 35B40. 
for small $\varepsilon>0$ we need to introduce corrections to the solutions that ensure that the remaining $m-\mu$ boundary conditions are satisfied. Vishik and Lyusternik discovered that when an elliptic problem degenerates into an elliptic problem of smaller order, these corrections decrease outside a $\delta$-neighborhood of the boundary as $\exp \{-C(\delta) / \varepsilon\}$. Such corrections are usually called exponential boundary layers. Vishik and Lyusternik suggested a simple explicit method to construct the boundary layer; the method is based on solving a boundary value problem on the half-line for an ordinary differential equation with respect to the operator of differentiation in the direction transveral to the boundary. This method was frequently used in applied problems.

As for purely mathematical papers related to [1], there are not too many of them. The so-called general theory of elliptic boundary problems was not as popular at the time [1] appeared as it was 10 years later. By the way, this explains why the authors restricted the exposition to strongly elliptic operators and the Dirichlet problem. Generalizations of the theory in [1] to boundary problems of general type are considered in several papers by Frank (see [2]); detailed references to other publications can be found in the books [3, 4].

At the same time general boundary problems were considered also in the papers [5, [6. 7] by S. A. Nazarov, who generalized the Vishik-Lyusternik method to a class of domains with nonsmooth boundary. However, Nazarov did not formulate explicitly the Shapiro-Lopatinskii conditions for the problems he considered, replacing them by a priori estimates for the corresponding problems for ordinary differential operators on the halfline.

Generalizations of the theory with a small parameter to pseudodifferential problems were considered in the papers [8, 9] by A. S. Demidov and [10] by G. I. Eskin. Some results about ellipic problems with a small parameter were obtained in the book 11 . by Grubb using Boutet de Monvel algebras. As we have already mentioned, a detailed bibliography can be found in the books $[3$, 4 by Frank.

0.2. The present paper is, to a significant extent, of a methodological nature. Here we construct a systematic theory of the problem (0.1), (0.2) as an elliptic problem with a (small) parameter. The proper ellipticity conditions for the operator (0.1), which include, in particular, the Vishik-Lyusternik condition of regular degeneracy, are discussed in detail. For the problem (0.1), (0.2) with a small parameter we formulate analogs of the Shapiro-Lopatinskii-type ellipticity condition and prove that they are equivalent to Nazarov's analytical conditions.

In priinciple, the theory of the problem (0.1), (0.2) can be reduced to the following three groups of questions:

(A) Necessary and sufficient conditions on the leading symbols of operators (0.3), (0.4) that guarantee the existence of a sharp two-sided estimate that is uniform as $\varepsilon \searrow 0$.

(B) Construction of a formal asymptotic solution (FAS) of the problem (0.1), (0.2).

(C) The rationale of the formal asymptotic solution (i.e., an estimate of the discrepancy between the solution of (0.1), (0.2) and a finite sum of the asymptotic series).

Our main goal is the study of questions of type (A). Let us explain the main difference between the problem (0.1), (0.2) with small $\varepsilon$ and the traditional problem, corresponding, e.g., to $\varepsilon=1$. In both cases the analysis is based on the so-called locality principle, which reduces a problem with variable coefficients in a bounded domain to problems with constant coefficients in "model" domains. For a bounded domain $M$ with a smooth boundary $\partial M$, model domains are the entire space $\mathbb{R}^{n}$ and the half-space $\mathbb{R}_{+}^{n}$. 
Choosing a point $x^{0} \in M$ and taking the leading term of the operator (0.1) at this point,

$$
A(\xi, \varepsilon):=A^{0}\left(x^{0}, \xi, \varepsilon\right)=\sum \varepsilon^{2 m-2 \mu-j} A_{2 m-j}^{0}\left(x^{0}, \xi\right),
$$

we obtain the so-called inner symbol at the point $x^{0}$. In general, this is a nonhomogeneous polynomial in parameters $\xi$ and $\varepsilon$, which becomes homogeneous if $\varepsilon$ is assigned the weight -1 . The small parameter ellipticity condition allows us to obtain an estimate of the operator $A(D, \varepsilon)$ in $\mathbb{R}^{n}$ with respect to special norms depending on the parameter.

Now let the point under consideration belong to the boundary $x^{0}=x^{\prime 0} \in \partial M$. Choose a coordinate system $x=\left(x^{\prime}, x_{n}\right), x^{\prime} \in \mathbb{R}^{n-1}$, such that locally the boundary is given by the equation $x_{n}=0$. Denoting by $\xi=\left(\xi^{\prime}, \xi_{n}\right)$ the dual variables, we define

$$
B_{j}(\xi, \varepsilon):=\varepsilon^{b_{j}-\beta_{j}} B_{j, b_{j}}^{0}\left(x^{\prime 0}, \xi\right)+\varepsilon^{b_{j}-\beta_{j}-1} B_{j, b_{j}-1}^{0}\left(x^{\prime 0}, \xi\right)+\cdots+B_{j, \beta_{j}}^{0}\left(x^{\prime 0}, \xi\right) .
$$

The boundary symbol at the point $x^{\prime 0}$ is defined as the following boundary value problem for the ordinary differential operator on the half-line:

$$
\begin{gathered}
A\left(\xi^{\prime}, D_{t}, \varepsilon\right) v(t)=0, \quad t>0, \\
\left.B_{j}\left(\xi^{\prime}, D_{t}, \varepsilon\right) v(t)\right|_{t=0}=\phi_{j}, \quad j=1, \ldots, m, \\
v(t) \rightarrow 0, \quad t \rightarrow+\infty
\end{gathered}
$$

depending on two parameters $\xi^{\prime}$ and $\varepsilon$.

The study of the boundary value problem (0.7)-(0.9) is preceded by the analysis of the behavior of the roots of the algebraic equation

$$
A\left(\xi^{\prime}, \tau, \varepsilon\right)=\sum \varepsilon^{2 m-2 \mu-j} A_{2 m-j}^{0}\left(x^{\prime 0}, \xi^{\prime}, \tau\right)=0 .
$$

The presence of two parameters $\left|\xi^{\prime}\right|$ and $\varepsilon$ makes this problem far from trivial. It was first studied by Vishik and Lyusternik. In Section 1 we present a solution of this problem that is free of some nonessential restrictions imposed in 1. The roots $\tau\left(\xi^{\prime}, \varepsilon\right)$ of equation (0.10) that belong to the upper half-plane of the complex plane are divided into two groups: as $\varepsilon \rightarrow 0$ there are $\mu$ roots of order $O(1)$ and $m-\mu$ roots of order $O(1 / \varepsilon)$ (the so-called boundary layer roots). The existence of these roots makes the study of the invertibility of the boundary symbol a serious problem; this problem is analyzed using the Vishik-Lyusternik method. An application of this method yields important additional assumptions that are essential for the problem. These assumptions reduce to the condition of unique solvability of the following three problems on the half-line: the original problem (0.7)-(0.9) for $\left|\xi^{\prime}\right| \neq 0, \varepsilon>0$, the problem corresponding to $\varepsilon=0$ and $\mu$ boundary conditions, and a new special problem with $m-\mu$ boundary conditions determined by the Vishik-Lyusternik method. The solutions to this latter problem yield the so-called boundary layer corrections.

The first of the above conditions shows that for $\left|\xi^{\prime}\right|>0, \varepsilon>0$ there exist $m$ linearly independent solutions $v_{1}\left(t, \xi^{\prime}, \varepsilon\right), \ldots, v_{m}\left(t, \xi^{\prime}, \varepsilon\right)$ of the equation (0.7) that satisfy (0.9) and the boundary conditions

$$
\left.B_{k}\left(\xi^{\prime}, D_{t}, \varepsilon\right) v_{j}(t)\right|_{t=0}=\delta_{k j}, \quad k, j=1, \ldots, m ;
$$

these solutions form the so-called fundamental system of solutions of the problem (0.7)(0.9). The description of the roots of the equation (0.10) implies that the solutions of this problem actually decrease as $t \rightarrow \infty$. Therefore, for the fundamental system of solutions, the integral norms

$$
\left(\int_{0}^{+\infty}\left|D_{t}^{\ell} v_{j}\left(t, \xi^{\prime}, \varepsilon\right)\right|^{2} d t\right)^{1 / 2}<\infty, \quad j=1, \ldots, m, \quad \ell=0,1,2, \ldots
$$


are bounded. In Section 2 we give explicit estimates of the left-hand sides of (0.12) as functions of the parameters $\left|\xi^{\prime}\right|, \varepsilon, j, \ell$. These estimates form an analytic foundation of the theory presented in this paper.

The complicated dependence of the solutions of the problem (0.7)-0.9) on parameters requires that in the function spaces under consideration we choose special norms depending on a small parameter $\varepsilon$. Presumably, such norms were first introduced by A. S. Demidov in [8, 9]. In Section 3 we introduce and study in detail the norms that are later used to obtain a priori bounds for our problem (0.1), (0.2).

In Section 4 we obtain the main result of this paper. For the problem (0.1), (0.2) in the half-space we prove the equivalence of the condition of small parameter ellipticity and the existence of two-sided a priori estimates in the norms introduced in Section 3.

The standard localization technique allows us to use two-sided estimates for operators with constant coefficients in the half-space to obtain estimates for operators with variable coefficients on a manifold with boundary. This technique is well developed, and we will not consider it here.

In the appendix we present a construction of a formal asymptotic solution of the problem in the half-space using the Vishik-Lyusternik method, i.e., we construct an expansion of the solution to our problem in the asymptotic series in $\varepsilon$.

0.3. Together with problems of type (0.1), (0.2) one can consider problems obtained by replacing the "small" parameter $\varepsilon$ with the "large" parameter $\lambda=1 / \varepsilon$ that runs over the lower half-plane of the complex plane:

$$
\begin{gathered}
\tilde{A}(x, D, \varepsilon) u(x)=f(x), \quad x \in M, \\
\tilde{B}_{j}\left(x^{\prime}, D, \varepsilon\right) u\left(x^{\prime}\right)=g_{j}\left(x^{\prime}\right), \quad x^{\prime} \in \partial M, \quad j=1, \ldots, m,
\end{gathered}
$$

where the operators in (0.13) and (0.14) depend polynomially on $\lambda$ :

$$
\begin{aligned}
\tilde{A}(x, D, \lambda) & :=A_{2 m}(x, D)+\lambda A_{2 m-1}(x, D)+\cdots+\lambda^{2 m-2 \mu} A_{2 \mu}(x, D), \\
\tilde{B}_{j}\left(x^{\prime}, D, \lambda\right) & :=B_{j, b_{j}}\left(x^{\prime}, D\right)+\lambda B_{j, b_{j}-1}\left(x^{\prime}, D\right)+\cdots+\lambda^{b_{j}-\beta_{j}} B_{j, \beta_{j}}\left(x^{\prime}, D\right) .
\end{aligned}
$$

In the case of boundary conditions independent of $\varepsilon$, the problems (0.13), (0.14) were studied in 13,14 .

Agranovich and Vishik [12] related the theory of the mixed problem for parabolic (in the sense of Petrovskii) equations to the theory of elliptic problems with a parameter. For $\mu=0$ the problem (0.13), (0.14) turns into the problem studied by Agranovich and Vishik. For $\mu>0$ the problem (0.13), (0.14) is closely related to the mixed problem for parabolic equations not resolved with respect to the highest derivative in $t$. This connection will be followed up in detail in another paper 11

M. S. Agranovich carefully read the manuscript of the paper and made a number of useful remarks. I use this opportunity to express my gratitude to him.

\section{SMALl PARAMETER ELLiptic POLYNOMials}

1.1. We will consider polynomials of the form

$$
A(\xi, \varepsilon):=\varepsilon^{2 m-2 \mu} A_{2 m}(\xi)+\varepsilon^{2 m-2 \mu-1} A_{2 m-1}(\xi)+\cdots+A_{2 \mu}(\xi)
$$

in variables $\xi=\left(\xi_{1}, \ldots, \xi_{n}\right) \in \mathbb{R}^{n}$ and $\varepsilon \in \mathbb{R}_{+}$. As in the introduction, $A_{2 m-j}(\xi)$ is a polynomial of order $2 m-j$ and by $A_{2 m-j}^{0}(\xi)$ we denote the leading homogeneous term of $A_{2 m-j}$. Replacing in (1.1) $A_{2 m-j}(\xi)$ with $A_{2 m-j}^{0}(\xi)$, we obtain the leading term of

\footnotetext{
${ }^{1}$ L. R. Volevich, The Vishik-Lyusternik method in parabolic equations unresolved with respect to the highest time derivative. Trans. Moscow Math. Soc. 68 (2007). Added in translation.
} 
$A^{0}(\xi, \varepsilon)$. Assigning to the parameter $\varepsilon$ weight -1 and to the variables $\xi$ weight 1 we see that $A^{0}(\xi, \varepsilon)$ is a quasihomogeneous polynomial of order $2 \mu$.

Definition 1.1. The polynomial (1.1) is said to be small parameter elliptic if its leading term $A^{0}(\xi, \varepsilon)$ admits a lower bound

$$
\left|A^{0}(\xi, \varepsilon)\right| \geq C|\xi|^{2 \mu}(1+\varepsilon|\xi|)^{2 m-2 \mu} .
$$

Let us note that (1.1) and the definition of $A^{0}(\xi, \varepsilon)$ imply that the estimate (1.2) is in fact two-sided. This takes place if and only if

$$
\left|G^{0}(\xi, \varepsilon)\right| \leq C^{-1}|\xi|^{-2 \mu}(1+\varepsilon|\xi|)^{-2 m+2 \mu}, \quad G^{0}(\xi, \varepsilon):=\left(A^{0}(\xi, \varepsilon)\right)^{-1} .
$$

Proposition 1.2. The polynomial (1.1) is small parameter elliptic if and only if the following conditions are satisfied:

(i) the polynomial $A_{2 m}(\xi)$ is elliptic, i.e., $A_{2 m}^{0}(\xi) \neq 0, \xi \neq 0$;

(ii) the polynomial $A_{2 \mu}(\xi)$ is elliptic, i.e., $A_{2 \mu}^{0}(\xi) \neq 0, \xi \neq 0$;

(iii) $A^{0}(\xi, \varepsilon) \neq 0,|\xi|>0, \varepsilon \geq 0$.

Proof. The necessity of (iii) follows immediately from (1.2). Setting in (1.2) $\varepsilon=0$, we prove the necessity of (ii). Dividing both parts of (1.2) by $\varepsilon^{2 m-2 \mu}$ and passing to the limit as $\varepsilon \rightarrow \infty$, we prove the necessity of (i).

Sufficiency. Using the ellipticity of the polynomial $A_{2 \mu}(\xi)$, we write

$$
A^{0}(\xi, \varepsilon):=A_{2 \mu}^{0}(\xi) \varepsilon^{2 m-2 \mu} C^{0}(\xi, \lambda), \quad \lambda:=\frac{1}{\varepsilon},
$$

where

$$
C^{0}(\xi, \lambda)=\frac{A_{2 m}(\xi)}{A_{2 \mu}(\xi)}+\lambda \frac{A_{2 m-1}(\xi)}{A_{2 \mu}(\xi)}+\cdots+\lambda^{2 m-2 \mu}
$$

By (iii),

$$
C^{0}(\xi, \lambda) \neq 0, \quad|\xi| \neq 0 .
$$

But $C^{0}(0, \lambda)=\lambda^{2 m-2 \mu} \neq 0$ for $\lambda>0$, i.e., the Agranovich-Vishik small parameter ellipticity condition is satisfied for $C^{0}(\xi, \lambda)$ :

$$
C^{0}(\xi, \lambda) \neq 0, \quad|\xi|+|\lambda|>0
$$

But then $C^{0}(\xi, \lambda)$ is estimated from above and from below in terms of $(|\lambda|+|\xi|)^{2 m-2 \mu}$, and we obtain inequality (1.2).

The above proof shows that together with polynomials with a "small" parameter $\varepsilon$ it is natural to consider polynomials with a "large" parameter $\lambda$ :

$$
\tilde{A}(\xi, \lambda):=A_{2 m}(\xi)+\lambda A_{2 m-1}(\xi)+\cdots+\lambda^{2 m-2 \mu} A_{2 \mu}(\xi) .
$$

Replacing the polynomials $A_{2 m}, \ldots, A_{2 \mu}$ with their leading terms $A_{2 m}^{0}, \ldots, A_{2 \mu}^{0}$, we obtain the leading term $\tilde{A}^{0}(\xi, \lambda)$ of the polynomial (1.3). Polynomials (1.1) and (1.3) are connected by the formula

$$
\tilde{A}\left(\xi, \varepsilon^{-1}\right)=\varepsilon^{-2 m+2 \mu} A(\xi, \varepsilon) .
$$

Definition 1.3. The polynomial (1.3) is said to be weakly parameter-elliptic if its leading term $\tilde{A}^{0}(\xi, \lambda)$ satisfies the following lower bound:

$$
\left|\tilde{A}^{0}(\xi, \lambda)\right| \geq C|\xi|^{2 \mu}(|\lambda|+|\xi|)^{2 m-2 \mu} .
$$

Formula (1.4) implies that the polynomial (1.3) is weakly elliptic with parameter $\lambda=1 / \varepsilon$ if and only if the polynomial (1.1) is elliptic with the small parameter $\varepsilon$.

For polynomials thar are weakly parameter-elliptic, an analogue of Proposition 1.2 holds; one only needs to replace $A^{0}(\xi, \varepsilon)$ with $\tilde{A}^{0}(\xi, \lambda)$. 
Remark 1.4. 1) From the homogeneity of $A^{0}$ it follows that without the loss of generality we can assume condition (iii) to hold only for $\varepsilon=1$.

2) The condition of small parameter ellipticity essentially appeared in the VishikLyusternik paper [1]. The condition of weak parameter ellipticity was introduced in [13, 14]. For $\mu=0$ it becomes the Agranovich-Vishik parameter-ellipticity condition.

1.2. Remarks about roots of small parameter elliptic polynomials. Set $\xi=$ $\left(\xi^{\prime}, \xi_{n}\right), \xi^{\prime}=\left(\xi_{1}, \ldots, \xi_{n-1}\right)$. In studying boundary value problems an important role is played by the roots of the algebraic equation

$$
A^{0}\left(\xi^{\prime}, \tau, \varepsilon\right)=0, \quad\left(\xi^{\prime}, \varepsilon\right) \in \mathbb{R}^{n-1} \times \mathbb{R}_{+},
$$

belonging to the upper half-plane $\mathbb{C}_{+}$of the complex plane.

However, we start with the study of the dependence of all roots of the equation (1.5) on $\varepsilon$. For $\varepsilon \neq 0$ this equation can be rewritten as follows:

$$
\tilde{A}^{0}\left(\xi^{\prime}, \tau, 1 / \varepsilon\right)=0, \quad\left(\xi^{\prime}, \varepsilon\right) \in \mathbb{R}^{n-1} \times \mathbb{R}_{+} .
$$

The roots of this equation depend continuously on the parameter $1 / \varepsilon$ outside of an arbitrarily small neighborhood of the point $\varepsilon=0$, and we can choose, in general nonuniquely, $2 m$ continuous branches $\tau_{j}\left(\xi^{\prime}, \varepsilon\right), j=1, \ldots, 2 m$. Due to the invariance of our equation with respect to the substitutions $\left(\xi^{\prime}, \tau, \varepsilon\right) \rightarrow\left(r \xi^{\prime}, r \tau, \varepsilon / r\right)$ we can assume that the branches are selected so that

$$
\tau_{j}\left(\xi^{\prime}, \varepsilon\right)=\frac{1}{r} \tau_{j}\left(r \xi^{\prime}, \varepsilon / r\right) \quad \forall r>0, \quad j=1, \ldots, 2 m .
$$

Our goal is to study the behavior, as $\varepsilon \rightarrow 0$, of branches satisfying (1.7). We will need certain notation.

By $S\left(\xi^{\prime}, \varepsilon\right)$ and $S_{2 \mu}\left(\xi^{\prime}\right)$ we denote respectively the set of complex roots of equation (1.5) and of the equation

$$
A_{2 \mu}^{0}\left(\xi^{\prime}, \tau\right)=0,
$$

which becomes (1.5) at $\varepsilon=0$.

In studying the behavior of the set $S\left(\xi^{\prime}, \varepsilon\right)$ for small $\varepsilon$, a fundamental role is played by the roots of the following polynomial, introduced by Vishik and Lyusternik:

$$
Q(\tau):=\frac{A^{0}(0, \tau, 1)}{A_{2 \mu}^{0}(0, \tau)}=\sum_{j=0}^{2 m-2 \mu} q_{j} \tau^{2 m-2 \mu-j}, \quad q_{j}=\frac{A_{2 m-j}^{0}(0,1)}{A_{2 \mu}^{0}(0,1)} .
$$

We denote by $S_{\mathrm{blr}}$ the set of all complex roots of the polynomial (1.9). We show that for sufficiently small $\varepsilon$ the set $S\left(\xi^{\prime}, \varepsilon\right)$ splits into two disjoint subsets

$$
S\left(\xi^{\prime}, \varepsilon\right)=S^{1}\left(\xi^{\prime}, \varepsilon\right) \cup S^{2}\left(\xi^{\prime}, \varepsilon\right),
$$

where

$$
S^{1}\left(\xi^{\prime}, \varepsilon\right) \rightarrow S_{2 \mu}\left(\xi^{\prime}\right), \quad \varepsilon S^{2}\left(\xi^{\prime}, \varepsilon\right) \rightarrow S_{\mathrm{blr}}, \quad \varepsilon \rightarrow 0 .
$$

in the set-theoretic sense. Following [1], the roots in the second subset in (1.10) are called boundary layer roots.

More precisely, we have the following result.

Proposition 1.5. Let the polynomial $A\left(\xi^{\prime}, \tau, \varepsilon\right)$ be of the form (1.1). For an appropriate numbering of the roots $\tau_{j}\left(\xi^{\prime}, 1 / \varepsilon\right)$ of equation (1.6) the sets

$$
S^{1}\left(\xi^{\prime}, \varepsilon\right)=\left\{\tau_{1}\left(\xi^{\prime}, \varepsilon\right), \ldots, \tau_{2 \mu}\left(\xi^{\prime}, \varepsilon\right)\right\}, \quad S^{2}\left(\xi^{\prime}, \varepsilon\right)=\left\{\tau_{2 \mu+1}\left(\xi^{\prime}, \varepsilon\right), \ldots, \tau_{2 m}\left(\xi^{\prime}, \varepsilon\right)\right\}
$$


have the following properties:

(i) For any $\delta>0$ there exists $\varepsilon_{0}>0$ such that for $\varepsilon \leq \varepsilon_{0}$ the distance between the sets $S^{1}\left(\xi^{\prime}, \varepsilon\right)$ and $S_{2 \mu}\left(\xi^{\prime}\right)$ is less than $\delta\left|\xi^{\prime}\right|$.

(ii) For any $\delta>0$ there exists $\varepsilon_{0}>0$ such that for $\varepsilon \leq \varepsilon_{0}$ the distance between the sets $\varepsilon S^{1}\left(\xi^{\prime}, \varepsilon\right)$ and $S_{\mathrm{blr}}$ is less than $\delta\left|\xi^{\prime}\right|^{\kappa}$ for some $\kappa>0$.

This result is essentially contained in [1], and in the present formulation it is proved in [13. For completeness, we sketch the proof here.

Proof. Without loss of generality, we can assume that $\xi^{\prime}$ belongs to the unit sphere, i.e., $\xi^{\prime}=\omega$, where $|\omega|=1$. Indeed, setting in (1.7) $r=1 /\left|\xi^{\prime}\right|$, we obtain

$$
\tau_{j}\left(\xi^{\prime}, \varepsilon\right)=\left|\xi^{\prime}\right| \tau_{j}\left(\omega, \varepsilon\left|\xi^{\prime}\right|\right), \quad j=1, \ldots, m .
$$

For a fixed $\omega$, let a root $\tau_{j}=\cdots=\tau_{j+p-1}$ have multiplicity $p$. Then there exists $\alpha=\alpha(\omega)>0$ such that the equality

$$
\frac{1}{2 \pi i} \int_{\left|z-\tau_{j}\right|=\alpha} \frac{\frac{d}{d z} A(\omega, z, \varepsilon)}{A(\omega, z, \varepsilon)} d z=\frac{1}{2 \pi i} \int_{\left|z-\tau_{j}\right|=\alpha} \frac{\frac{d}{d z} A(\omega, z, 0)}{A(\omega, z, 0)} d z=p
$$

holds for all $\varepsilon<\varepsilon_{0}=\varepsilon_{0}(\omega)$. Therefore, for all $\varepsilon<\varepsilon_{0}$ the equation (1.5) has exactly $p$ roots in the disk $\left\{z \in \mathbb{C}:\left|z-\tau_{j}\right|<\alpha(\omega)\right\}$; we denote them by $\tau_{j}(\omega, \varepsilon), \ldots, \tau_{j+p-1}(\omega, \varepsilon)$. Applying this procedure to all roots of (1.5), for a fixed $\omega$ we obtain the set of roots $S^{1}(\omega, \varepsilon):=\left\{\tau_{1}(\omega, \varepsilon), \ldots, \tau_{2 \mu}(\omega, \varepsilon)\right\}$ of equation (1.5), which tends to $S_{2 \mu}(\omega)$ in the settheoretic sense. It remains to verify that the convergence is uniform over the points $\omega$ of the unit sphere.

Arguing by contradiction let us assume that statement (i) does not hold. Then there exists a sequence $\left(\omega_{n}\right)_{n \geq 1},\left|\omega_{n}\right|=1$, and a constant $C>0$ such that

$$
\operatorname{dist}\left(S_{2 \mu}\left(\omega_{n}\right), S^{1}\left(\omega_{n}, \varepsilon_{n}\right)\right) \geq C \quad \forall n \geq 1, \quad \varepsilon_{n}=\frac{1}{n} .
$$

By compactness, we can assume that the sequence $\omega_{n}$ converges to a point $\omega_{0}$. Since the roots of $A_{2 \mu}(\omega, \tau)$ continuously depend on $\omega$, for large $n$ we obtain

$$
\operatorname{dist}\left(S_{2 \mu}\left(\omega_{0}\right), S\left(\omega_{n}, \varepsilon_{n}\right)\right) \geq \frac{C}{2} .
$$

However, the above arguments imply that for a sufficiently small $\alpha>0$ there exist $\varepsilon_{0}=\varepsilon_{0}\left(\omega_{0}\right)$ and $s>0$ such that $A^{0}(\omega, \tau, \varepsilon)$ has precisely $2 \mu$ roots in $\bigcup_{j}\{z \in \mathbb{C}$ : $\left.\left|z-\tau_{j}\left(\omega_{0}\right)\right|<\alpha\right\}$ for all $\left|\omega-\omega_{0}\right|<s$ and $0<\varepsilon<\varepsilon_{0}$. Taking $\alpha<\frac{C}{2}$, we obtain a contradiction with (1.12).

(ii) Introduce the notation

$$
\xi^{\prime}=\rho \omega, \quad|\omega|=1, \quad \varepsilon=\frac{\tilde{\varepsilon}}{\rho}, \quad \tau=\frac{\tilde{\tau}}{\varepsilon}=\frac{\rho \tilde{\tau}}{\tilde{\varepsilon}} .
$$

Taking into account (1.1), we obtain

$$
A^{0}\left(\xi^{\prime}, \tau, \varepsilon\right)=\left(\frac{\rho}{\tilde{\varepsilon}}\right)^{2 \mu} A^{0}(\tilde{\varepsilon} \omega, \tilde{\tau}, 1)
$$

and the equation (1.5) turns into an equivalent equation

$$
A^{0}(\tilde{\varepsilon} \omega, \tilde{\tau}, 1)=A_{2 \mu}^{0}(0,1) \tilde{\tau}^{2 \mu} Q(\tilde{\tau})+\sum_{k=1}^{2 m} \tilde{\varepsilon}^{k} a_{k}(\omega, \tilde{\tau})=0,
$$

where the coefficients

$$
a_{k}(\omega, \tilde{\tau}):=\left.\frac{1}{k !}\left(\frac{\partial}{\partial \varepsilon}\right)^{k} A^{0}(\varepsilon \omega, \tilde{\tau}, 1)\right|_{\varepsilon=0}
$$

are smooth functions of $\omega$. 
Let $\tau_{j}^{1}=\cdots=\tau_{j+p-1}^{1}$ be a multiplicity $p$ root of the polynomial $Q(\tau)$. It is well known from the theory of algebraic functions that there exists a multi-valued analytic function (the Puiseux series)

$$
\tilde{\tau}(\omega, \varepsilon)=\tau_{j}^{1}+\sum_{k=1}^{\infty} c_{j k}(\omega) \tilde{\varepsilon}^{k / p} .
$$

This function has $p$ branches (corresponding to the branches of the function $\tilde{\varepsilon}^{\frac{1}{p}}$ ) that are solutions of the equation (1.13). We will denote the branches of the function (1.14) by $\tilde{\tau}_{s}(\omega, \varepsilon)$, where $s=j, \ldots, j+p-1$.

For a fixed $\omega$ and $|\tilde{\varepsilon}| \leq \tilde{\varepsilon}_{1}(\omega)$ this series is absolutely convergent. Explicit construction of the Puiseux series (see [15]) easily implies that the coefficients $c_{j k}(\omega)$ of the series (1.14) are continuous functions in $\omega$. Therefore, there exists $\tilde{\varepsilon}_{1}>0$ independent of $\omega$ and such that the series (1.14) is a holomorphic function in $\tilde{\varepsilon}^{\frac{1}{p}}$ for $|\tilde{\varepsilon}| \leq \tilde{\varepsilon}_{1}$.

Returning to the original variables $\tau, \varepsilon, \xi^{\prime}$ we obtain the formula

$$
\tau_{j}\left(\xi^{\prime}, \varepsilon\right)=\frac{\tau_{j}^{1}}{\varepsilon}+\tau_{j}^{2}\left(\xi^{\prime}, \varepsilon\right), \quad j=2 \mu+1, \ldots, 2 m,
$$

where

$$
\tau_{j}^{2}\left(\xi^{\prime}, \varepsilon\right)=\varepsilon^{-1} \sum_{k=1}^{\infty} c_{j k}(\omega) \tilde{\varepsilon}^{k / p}=\varepsilon^{-1+\frac{1}{p}}\left|\xi^{\prime}\right|^{\frac{1}{p}} \sum_{k=1}^{\infty} c_{j k}(\omega) \tilde{\varepsilon}^{(k-1) / p} .
$$

The convergence of the series implies that

$$
\left|\varepsilon \tau_{j}^{2}\left(\xi^{\prime}, \varepsilon\right)-\tau_{j}^{1}\right| \leq K_{j} \varepsilon^{\frac{1}{k_{1}}}\left|\xi^{\prime}\right|^{\frac{1}{k_{1}}},
$$

where $k_{1}$ is the maximum multiplicity of the roots of the polynomial $Q(\tau)$, thus proving (ii).

1.3. Proper small parameter elliptic polynomials. Let $m^{+}\left(\xi^{\prime}, \varepsilon\right)$ and $m^{-}\left(\xi^{\prime}, \varepsilon\right)$ be the number of solutions of the equation (1.6) in the open upper (resp. lower) halfplane. If the polynomial (1.1) is small parameter elliptic, then the equation (1.6) has no real solutions and the numbers $m^{ \pm}\left(\xi^{\prime}, \varepsilon\right)$ do not depend on $\xi^{\prime}$ and $\varepsilon$; we denote these numbers by $m^{ \pm}$. The absence of real solutions also implies that $m^{+}+m^{-}=2 m$. Since the solutions of (1.6) continuously depend on $\varepsilon$ in a neighborhood of $\varepsilon=\infty$, the numbers $m^{ \pm}$coincide with the number of solutions of the equation $A_{2 m}^{0}\left(\xi^{\prime}, \tau\right)=0$ in the upper (resp. lower) half-plane.

By $\mu^{ \pm}$and $q^{ \pm}$we denote the number of the corresponding solutions of the equation (1.8) and of the equation $Q(\tau)=0$ respectively.

If the condition of small parameter ellipticity is satisfied, then setting in (1.2) $\xi^{\prime}=0$ and dividing both sides of the inequality by $\tau^{2 \mu}$, we obtain

$$
|Q(\tau)|>C(1+\varepsilon|\tau|)^{2 m-2 \mu}
$$

This implies that the polynomial $Q(\tau)$ has no real roots, so that

$$
q^{+}+q^{-}=2 m-2 \mu \text {. }
$$

Since the polynomial $A_{2 \mu}^{0}$ is elliptic, it has no real roots for $\xi^{\prime} \neq 0$. Therefore, taking into account Proposition [1.5, we obtain

$$
m^{+}=\mu^{+}+q^{+}, \quad m^{-}=\mu^{-}+q^{-} .
$$

Definition 1.6. The polynomial (1.1) is said to be properly small parameter elliptic if

$$
m^{+}=m^{-}=m, \quad \mu^{+}=\mu^{-}=\mu .
$$


The condition of proper ellipticity (1.17) and the equalities (1.16) imply that

$$
q^{+}=q^{-}=m-\mu .
$$

Due to the condition of small parameter ellipticity the above sets of roots split into disjoint sets of roots lying in the upper (resp., lower) half-space of the complex plane. Therefore, we have

$$
\begin{gathered}
S\left(\xi^{\prime}, \varepsilon\right)=S^{+}\left(\xi^{\prime}, \varepsilon\right) \cup S^{-}\left(\xi^{\prime}, \varepsilon\right), \\
S^{1}\left(\xi^{\prime}, \varepsilon\right)=S^{1+}\left(\xi^{\prime}, \varepsilon\right) \cup S^{1-}\left(\xi^{\prime}, \varepsilon\right), \quad S_{2 \mu}\left(\xi^{\prime}\right)=S_{2 \mu}^{+}\left(\xi^{\prime}\right) \cup S_{2 \mu}^{-}\left(\xi^{\prime}\right), \\
S^{2}\left(\xi^{\prime}, \varepsilon\right)=S^{2+}\left(\xi^{\prime}, \varepsilon\right) \cup S^{2-}\left(\xi^{\prime}, \varepsilon\right), \quad S_{\mathrm{blr}}=S_{\mathrm{blr}}^{+} \cup S_{\mathrm{blr}}^{-} .
\end{gathered}
$$

Proposition 1.5 implies that

$$
S^{1+}\left(\xi^{\prime}, \varepsilon\right) \rightarrow S_{2 \mu}^{+}\left(\xi^{\prime}\right), \quad \varepsilon S^{2+}\left(\xi^{\prime}, \varepsilon\right) \rightarrow S_{\mathrm{blr}}^{+}, \quad \varepsilon \rightarrow 0 .
$$

More precisely, we have

Proposition 1.7. Let the polynomial (1.1) be properly small parameter elliptic. Then for an appropriate numbering of the solutions $\tau_{j}^{+}\left(\xi^{\prime}, 1 / \varepsilon\right)$ of equation (1.6) in the upper complex half-plane, the sets

$$
S^{1+}\left(\xi^{\prime}, \varepsilon\right)=\left\{\tau_{1}^{+}\left(\xi^{\prime}, \varepsilon\right), \ldots, \tau_{\mu}^{+}\left(\xi^{\prime}, \varepsilon\right)\right\}, \quad S^{2+}\left(\xi^{\prime}, \varepsilon\right)=\left\{\tau_{\mu+1}^{+}\left(\xi^{\prime}, \varepsilon\right), \ldots, \tau_{m}^{+}\left(\xi^{\prime}, \varepsilon\right)\right\}
$$

have the following properties:

(i) For an arbitrary $\delta>0$ there exists $\varepsilon_{0}>0$ such that for $\varepsilon \leq \varepsilon_{0}$ the distance between the sets $S^{1+}\left(\xi^{\prime}, \varepsilon\right)$ and $S_{2 \mu}^{+}\left(\xi^{\prime}\right)$ is less than $\delta\left|\xi^{\prime}\right|$.

(ii) For an arbitrary $\delta>0$ there exists $\varepsilon_{0}>0$ such that for $\varepsilon \leq \varepsilon_{0}$ the distance between the sets $\varepsilon S^{2+}\left(\xi^{\prime}, \varepsilon\right)$ and $S_{\mathrm{blr}}^{+}$is less than $\delta\left|\xi^{\prime}\right|^{\kappa}$ for some $0<\kappa \leq 0$.

Remark 1.8. 1) It is well known that (1.17) is an additional restriction only for $n=2$ since for $n>2$ it is satisfied automatically.

2) Proposition 1.7 was first formulated and proved in [1] under the condition $q^{+}=$ $q^{-}=m-\mu$, which is called the condition for the regularity of degeneration. In [1] additional restrictions of a technical nature concerning the simplicity of the roots were used. The paper 13 contains a proof that does not use these restrictions; this proof is basically reproduced in the proofs of Propositions 1.5 and 1.7 of the present paper. A new feature of the present proof is that the regularity condition in [1] is a consequence of the condition of proper small parameter ellipticity.

1.4. Factorization of small parameter elliptic polynomials. The splitting of the solutions of (1.5) into two groups (Proposition 1.5) is related to the factorization of the corresponding polynomial (see [16, Proposition 1 in III.1.3]):

$$
A^{0}\left(\xi^{\prime}, \tau, \varepsilon\right)=A^{1}\left(\tau, \xi^{\prime}, \varepsilon\right) A^{2}\left(\tau, \xi^{\prime}, \varepsilon\right) .
$$

The factorization is determined uniquely up to a constant, and we normalize the first factor in (1.19) by setting

$$
A^{1}\left(\tau, \xi^{\prime}, \varepsilon\right)=A_{2 \mu}^{0}(1,0) \prod_{j=1}^{2 \mu}\left(\tau-\tau_{j}\left(\xi^{\prime}, \varepsilon\right)\right) .
$$

Proposition 1.5(i) implies that

$$
A^{1}\left(\tau, \xi^{\prime}, \varepsilon\right) \rightarrow A_{2 \mu}^{0}\left(\xi^{\prime}, \tau\right), \quad \varepsilon \rightarrow 0 .
$$

Now we prove that

$$
A^{2}\left(\frac{\tau}{\varepsilon}, \xi^{\prime}, \varepsilon\right) \rightarrow Q(\tau), \quad \varepsilon \rightarrow 0 .
$$


Taking into account (1.19), (1.20) we have

$$
A^{2}\left(\tau, \xi^{\prime}, \varepsilon\right)=\frac{A_{2 m}^{0}(1,0)}{A_{2 \mu}^{0}(1,0)} \varepsilon^{2 m-2 \mu} \prod_{j=2 \mu+1}^{2 m}\left(\tau-\tau_{j}\left(\xi^{\prime}, \varepsilon\right)\right),
$$

whence

$$
A^{2}\left(\frac{\tau}{\varepsilon}, \xi^{\prime}, \varepsilon\right)=\frac{A_{2 m}^{0}(1,0)}{A_{2 \mu}^{0}(1,0)} \prod_{j=2 \mu+1}^{2 m}\left(\tau-\varepsilon \tau_{j}\left(\xi^{\prime}, \varepsilon\right)\right) .
$$

Setting in (1.7) $r=\varepsilon$, we obtain that $\varepsilon \tau_{j}\left(\xi^{\prime}, \varepsilon\right)=\tau_{j}\left(\varepsilon \xi^{\prime}, 1\right)$. Therefore,

$$
A^{2}\left(\frac{\tau}{\varepsilon}, \xi^{\prime}, \varepsilon\right)=\frac{A_{2 m}^{0}(1,0)}{A_{2 \mu}^{0}(1,0)} \prod_{j=2 \mu+1}^{2 m}\left(\tau-\tau_{j}\left(\varepsilon \xi^{\prime}, 1\right)\right)=A^{2}\left(\tau, \varepsilon \xi^{\prime}, 1\right) .
$$

This formula and Proposition 1.5(ii) imply (1.22).

Proposition 1.7 yields the following additional factorization:

$$
\begin{aligned}
& A^{1}\left(\xi^{\prime}, \tau, \varepsilon\right)=A^{1+}\left(\tau, \xi^{\prime}, \varepsilon\right) A^{1-}\left(\tau, \xi^{\prime}, \varepsilon\right), \\
& A^{2}\left(\xi^{\prime}, \tau, \varepsilon\right)=A^{2+}\left(\tau, \xi^{\prime}, \varepsilon\right) A^{2-}\left(\tau, \xi^{\prime}, \varepsilon\right) .
\end{aligned}
$$

It turns out that

$$
A^{1+}\left(\tau, \xi^{\prime}, \varepsilon\right) \rightarrow A_{2 \mu}^{0+}\left(\xi^{\prime}, \tau\right), \quad A^{2+}\left(\tau / \varepsilon, \xi^{\prime}, \varepsilon\right) \rightarrow Q^{+}(\tau), \quad \varepsilon \rightarrow 0 .
$$

\section{Elliptic PRoblems With a SMAll PARAMETER IN THE HalF-SPACE}

In this section we define the notion of an elliptic problem with a small parameter in the half-space, which is the main definition of the present paper. Using the VishikLyusternik method, we construct a fundamental system of solutions for such a problem and find integral estimates for derivatives of solutions of the problem.

We start by recalling some notation already used in the introduction.

2.1. Main notation. We will consider the boundary symbol of the problem (0.1), (0.2) (already defined in the introduction) at a point of the boundary. This is the boundary problem on the half-line for the ordinary differential operator

$$
\begin{aligned}
A\left(\xi^{\prime}, D_{t}, \varepsilon\right) v(t) & =0, \quad t>0, \\
B_{j}\left(\xi^{\prime}, D_{t}, \varepsilon\right) v(0) & =\phi_{j}, \quad j=1, \ldots, m, \\
v(t) & \rightarrow 0, \quad t \rightarrow+\infty,
\end{aligned}
$$

depending on the parameters $\xi^{\prime}$ and $\varepsilon$. Here

$$
A(\xi, \varepsilon):=\varepsilon^{2 m-2 \mu} A_{2 m}(\xi)+\varepsilon^{2 m-2 \mu-1} A_{2 m-1}(\xi)+\cdots+A_{2 \mu}(\xi)
$$

is a polynomial in the variables $\xi=\left(\xi^{\prime}, \xi_{n}\right)$, and the polynomials $A_{2 m-k}$ coincide with their leading terms, i.e., are homogeneous functions of degree $2 m-k$. Similarly,

$$
B_{j}(\xi, \varepsilon)=\varepsilon^{b_{j}-\beta_{j}} B_{j, b_{j}}\left(x^{\prime 0}, \xi\right)+\varepsilon^{b_{j}-\beta_{j}-1} B_{j, b_{j}-1}\left(x^{\prime 0}, \xi\right)+\cdots+B_{\beta_{j}}\left(x^{\prime 0}, \xi\right),
$$

where $B_{j, b_{j}-k}$ are homogeneous functions of degree $b_{j}-k$.

We will assume that the sequence $\beta_{j}$ is nondecreasing; moreover,

$$
\beta_{1} \leq \beta_{2} \leq \cdots \leq \beta_{\mu}<\beta_{\mu+1} \leq \cdots \leq \beta_{m}
$$

In the standard theory of solvability of elliptic (or parameter-elliptic) problems one obtains various forms of the invertibility condition for operators of the form (2.1); this condition is called the Shapiro-Lopatinskii condition (or the Agmon condition, or the Agranovich-Vishik condition). In our case this condition is too weak and does not allow 
us to obtain two-sided estimates for solutions that would be uniform with respect to the small parameter.

Before moving further we recall some known facts about the Shapiro-Lopatinskii condition.

2.2. Some comments about the Shapiro-Lopatinskii condition. We start with simple facts about the following problem for an ordinary differential operator on the half-line:

$$
\begin{aligned}
A\left(D_{t}\right) v(t) & =0, \quad t>0, \\
B_{j}\left(D_{t}\right) v(0) & =g_{j}, \quad j=1, \ldots, \nu, \\
v(t) & \rightarrow 0, \quad t \rightarrow+\infty .
\end{aligned}
$$

Here $A(\tau)$ is a complex polynomial without real roots and with $\nu$ roots in the upper half-plane. Denoting these roots by $\tau_{1}^{+}, \ldots, \tau_{\nu}^{+}$, we define

$$
A^{+}(\tau):=\prod_{j=1}^{\nu}\left(\tau-\tau_{j}^{+}\right) .
$$

Let $B_{1}(\tau), \ldots, B_{\nu}(\tau)$ be polynomials of degrees $m_{1}, \ldots, m_{\nu}$ respectively and let

$$
\bar{B}_{k}(\tau)=\sum_{i=1}^{\nu} \bar{b}_{i k} \tau^{i-1}
$$

be the remainder of the division of $B_{k}(\tau)$ by $A^{+}(\tau)$. The matrix

$$
\operatorname{Lop}\left(A, B_{1}, \ldots, B_{\nu}\right):=\left(\bar{b}_{i k}\right)_{i, k=1, \ldots, \nu}
$$

is called the Lopatinskii matrix.

The following result is well known in the theory of elliptic boundary value problems.

Lemma 2.1. The following conditions are equivalent:

(i) The problem (2.5) has a unique solution for each $\left(g_{1}, \ldots, g_{\nu}\right) \in \mathbb{C}^{\nu}$.

(ii) The Lopatinskii matrix $\operatorname{Lop}\left(A, B_{1}, \ldots, B_{\nu}\right)$ is invertible.

(iii) Let $\gamma$ be a closed contour in $\mathbb{C}_{+}$encircling all roots $\tau_{1}^{+}, \ldots, \tau_{\nu}^{+}$. Then there exist polynomials $N_{1}(\tau), \ldots, N_{\nu}(\tau)$ of degree less than $\nu$ such that

$$
\frac{1}{2 \pi i} \int_{\gamma} \frac{B_{i}(\tau) N_{k}(\tau)}{A_{+}(\tau)} d \tau=\delta_{i k}, \quad i, k=1, \ldots, \nu .
$$

Proof. (i) $\rightarrow$ (ii) follows from the fact that each solution of (2.5) is in fact a solution of the problem

$$
\begin{aligned}
A^{+}\left(D_{t}\right) v(t) & =0, \quad t>0, \\
\bar{B}_{j}\left(D_{t}\right) v(0) & =g_{j}, \quad j=1, \ldots, \nu, \\
v(t) & \rightarrow 0, \quad t \rightarrow+\infty .
\end{aligned}
$$

(iii) $\rightarrow$ (i). First of all note that the function

$$
v(t)=\frac{1}{2 \pi i} \sum_{j=1}^{\nu} g_{j} \int_{\gamma} e^{i \tau t} \frac{N_{j}(\tau)}{A_{\gamma}^{+}(\tau)} d \tau
$$

is a solution of the problem (2.5). Since the problem (2.5) is finite-dimensional, the solvability of the problem for arbitrary right-hand sides $\left(g_{1}, \ldots, g_{m}\right) \in \mathbb{C}^{\nu}$ implies the uniqueness of the solution. 
(ii) $\rightarrow$ (iii). We use the explicit construction of the polynomials $N_{1}(\tau), \ldots, N_{\nu}(\tau)$ presented in [17. We need the following notation:

$$
A^{+}(\tau):=\sum_{p=0}^{\nu} a_{p}^{+} \tau^{\nu-p}, \quad A_{j}^{+}(\tau):=\sum_{p=0}^{j} a_{p}^{+} \tau^{j-p}, \quad j=0, \ldots, \nu-1 .
$$

Denote by $\left(\bar{b}^{i k}\right)_{i, k=1, \ldots, \nu}$ the inverse to the Lopatinskii matrix. Then the polynomials

$$
N_{j}(\tau)=\sum_{i=1}^{\nu} \bar{b}^{i j} A_{\nu-i}^{+}(\tau)
$$

satisfy (2.6) (for the proof, see [17]).

Definition 2.2. A family of operators $\left\{A, B_{1}, \ldots, B_{\nu}\right\}$ satisfies the Shapiro-Lopatinskii condition if the equivalent conditions of Lemma 2.1 are satisfied.

Together with the original problems satisfying the Shapiro-Lopatinskii condition we will also consider small perturbations of these problems. This consideration is based on the following lemma.

Lemma 2.3. Let the operators $\left(A^{0}\left(D_{t}\right), B_{1}^{0}\left(D_{t}\right), \ldots, B_{\nu}^{0}\left(D_{t}\right)\right)$ determine the boundary value problem on the half-line of the form (2.5) that satisfies the equivalent conditions of Lemma 2.1. Denote by $\tau_{1}^{0+}, \ldots, \tau_{\nu}^{0+}$ the roots of the polynomial $A^{0}$ in $\mathbb{C}_{+}$and write

$$
B_{j}^{0}(\tau)=\sum_{\ell=0}^{m_{j}} b_{j \ell}^{0} \tau^{\ell} .
$$

Choose a contour $\gamma_{0} \subset \mathbb{C}_{+}$encircling $\tau_{1}^{0+}, \ldots, \tau_{\nu}^{0+}$ and denote by $N_{1}^{0}, \ldots, N_{\nu}^{0}$ the polynomials satisfying the conditions

$$
\frac{1}{2 \pi i} \int_{\gamma_{0}} \frac{B_{k}^{0}(\tau) N_{\ell}^{0}(\tau)}{A_{+}^{0+}(\tau)} d \tau=\delta_{k \ell}, \quad k, \ell=1, \ldots, \nu,
$$

where $A_{+}^{0+}(\tau)=\prod_{j=1}^{\nu}\left(\tau-\tau_{j}^{0+}\right)$. Then there exists $\delta>0$ with the following properties.

Let

$$
A^{+}(\tau)=\prod_{j=1}^{m}\left(\tau-\tau_{j}^{+}\right), \quad B_{j}(\tau)=\sum_{\ell=0}^{m_{j}} b_{j \ell} \tau^{\ell}
$$

be such that

$$
\begin{aligned}
\left|\tau_{j}^{+}-\tau_{j}^{0+}\right|<\delta, & j=1, \ldots, \nu, \\
\left|b_{j \ell}-b_{j \ell}^{0}\right|<\delta, & j=1, \ldots, \nu, \quad \ell=1, \ldots, m_{j} .
\end{aligned}
$$

Then the contour $\gamma_{0}$ encircles $\tau_{1}^{+}, \ldots, \tau_{\nu}^{+}$and there exist polynomials $N_{1}, \ldots, N_{\nu}$ such that

$$
\frac{1}{2 \pi i} \int_{\gamma^{0}} \frac{B_{k}(\tau) N_{\ell}(\tau)}{A^{+}(\tau)} d \tau=\delta_{k \ell}, \quad k, \ell=1, \ldots, \nu
$$

Proof. If $\delta>0$ is sufficiently small, the contour $\gamma_{0}$ encircles $\tau_{1}^{+}, \ldots, \tau_{\nu}^{+}$. By Lemma 2.1 the existence of polynomials $N_{1}, \ldots, N_{\nu}$ is equivalent to the invertibility of the Lopatinskii matrix $\operatorname{Lop}\left(A, B_{1}, \ldots, B_{\nu}\right):=\left(\bar{b}_{i k}\right)_{i, k=1, \ldots, m}$. To prove the invertibility of this matrix it suffices to note that its entries depend continuously on the coefficients of $B_{j}$ and $A^{+}$. The proof of Lemma 2.1 shows that the coefficients of the polynomials $N_{\ell}$ also depend continuously on the coefficients of $B_{j}$ and $A^{+}$, so that $N_{\ell}(\tau) \rightarrow N_{\ell}^{0}(\tau)$ as $\delta \rightarrow 0$. 
In problems of type (2.5) we are dealing with all solutions of the equation $A(\tau)=0$ in $\mathbb{C}_{+}$. Later we will consider questions involving only a group of such solutions. The naturality of these questions is explained by the factorization in 1.4. Let us give the corresponding definitions.

Suppose that a contour $\gamma \subset \mathbb{C}_{+}$does not pass through the roots of an algebraic equation $A(\tau)=0$ and encircles exactly $\nu$ roots (counting multiplicities). Denote by $\mathfrak{M}_{\gamma}$ the subspace of those solutions of the equation

$$
A\left(D_{t}\right) v(t)=0, \quad t>0,
$$

that can be represented in the form

$$
v(t)=\frac{1}{2 \pi i} \int_{\gamma} e^{i \tau t} \frac{C(\tau)}{A(\tau)} d \tau,
$$

where $C(\tau)$ is a polynomial of degree smaller than the degree of $A(\tau)$. One can easily see that $\operatorname{dim} \mathfrak{M}_{\gamma}=\nu$. Appending the equation with the boundary conditions at $t=0$, we obtain the following generalization of the problem (2.5):

$$
\begin{aligned}
A\left(D_{t}\right) v(t) & =0, \quad t>0, \\
B_{j}\left(D_{t}\right) v(0) & =g_{j}, \quad j=1, \ldots, \nu, \\
v(t) & \in \mathfrak{M}_{\gamma} .
\end{aligned}
$$

Denoting by $\tau_{1}^{+}, \ldots, \tau_{\nu}^{+}$the roots inside $\gamma$, we set

$$
A_{\gamma}^{+}(\tau):=\prod_{j=1}^{\nu}\left(\tau-\tau_{j}^{+}\right)
$$

Let

$$
\bar{B}_{k \gamma}(\tau):=\sum_{i=1}^{\nu} b_{i k \gamma} \tau^{i-1}
$$

be the remainder of the division of $B_{k}(\tau)$ by $A_{\gamma}^{+}(\tau)$. The matrix

$$
\operatorname{Lop}_{\gamma}\left(A, B_{1}, \ldots, B_{\nu}\right):=\left(\bar{b}_{i k \gamma}\right)_{i, k=1, \ldots, \nu}
$$

is called the Lopatinskii matrix associated with the contour $\gamma$.

The problem (2.8) admits direct analogs of Lemmas 2.1] and 2.3 .

Lemma 2.4. The following conditions are equivalent:

(i) The problem (2.8) has a unique solution for each $\left(g_{1}, \ldots, g_{\nu}\right) \in \mathbb{C}^{\nu}$.

(ii) The Lopatinskii matrix $\operatorname{Lop}_{\gamma}\left(A, B_{1}, \ldots, B_{\nu}\right)$ is invertible.

(iii) There exist polynomials $N_{1 \gamma}(\tau), \ldots, N_{\nu \gamma}(\tau)$ of degree less than $\nu$ such that

$$
\frac{1}{2 \pi i} \int_{\gamma} \frac{B_{i}(\tau) N_{k \gamma}(\tau)}{A_{\gamma}^{+}(\tau)} d \tau=\delta_{i k}, \quad i, k=1, \ldots, \nu .
$$

The proof is similar to the proof of Lemma 2.1, and we leave it to the reader. We also leave to the reader a trivial reformulation of Lemma 2.3 .

\subsection{Elliptic boundary value problems with a small parameter.}

Definition 2.5. The problem (0.1), (0.2) is called a small parameter elliptic problem if the following conditions are satisfied.

Condition (A). The polynomial (1.1) corresponding to the inner symbol at a generic point $x^{0} \in M$ satisfies the small parameter ellipticity condition. Moreover, for each point $x^{0} \in \partial M$ the corresponding polynomial (2.2) satisfies the proper small parameter ellipticity condition. 
Condition (B). The problem (2.1) corresponding to the boundary symbol of (0.1), (0.2) satisfies the Shapiro-Lopatinskii condition for each $\left|\xi^{\prime}\right|>0, \varepsilon>0$ (Lemma 2.1).

The next two conditions deal with the solvability of problems without a parameter obtained from (2.1) for small and for large $\varepsilon$. In the case $\varepsilon \rightarrow \infty$ we have the problem obtained from (2.1) by removing the lower terms:

$$
\begin{aligned}
A_{2 m}\left(\xi^{\prime}, D_{t}, \varepsilon\right) v(t) & =0, \quad t>0, \\
B_{j, b_{j}}\left(\xi^{\prime}, D_{t}, \varepsilon\right) v(0) & =\phi_{j}, \quad j=1, \ldots, m, \\
v(t) & \rightarrow 0, \quad t \rightarrow+\infty .
\end{aligned}
$$

For $\varepsilon=0$ the problem (2.1) becomes overdetermined since the resulting operator requires $\mu<m$ boundary conditions. Taking the first $\mu$ boundary conditions, we obtain the problem

$$
\begin{aligned}
A_{2 \mu}\left(\xi^{\prime}, D_{t}, \varepsilon\right) v(t) & =0, \quad t>0, \\
B_{j, \beta_{j}}\left(\xi^{\prime}, D_{t}, \varepsilon\right) v(0) & =\phi_{j}, \quad j=1, \ldots, \mu, \\
v(t) & \rightarrow 0, \quad t \rightarrow+\infty .
\end{aligned}
$$

Condition $\left(\mathrm{C}_{1}\right)$. The problem (2.10) is uniquely solvable, i.e., the operators $A_{2 m}, B_{b_{1}}$, $\ldots, B_{b_{m}}$ satisfy the Shapiro-Lopatinskii condition.

Condition $\left(\mathrm{C}_{2}\right)$. The problem (2.11) is uniquely solvable, i.e., the operators $A_{2 \mu}, B_{\beta_{1}}$, $\ldots, B_{\beta_{m}}$ satisfy the Shapiro-Lopatinskii condition (it is assumed that the condition (2.4) holds).

Finally, the Vishik-Lyusternik method suggests the following.

Condition $\left(\mathrm{C}_{3}\right)$. The operators $A\left(0, D_{t}, 1\right), B_{\mu+1}\left(0, D_{t}, 1\right), \ldots, B_{m}\left(0, D_{t}, 1\right)$ satisfy the Shapiro-Lopatinskii condition.

Let conditions (A) and (B) hold. Then for $\left|\xi^{\prime}\right|>0, \varepsilon>0$ there exist $m$ linearly independent solutions $v_{1}\left(t, \xi^{\prime}, \varepsilon\right), \ldots, v_{m}\left(t, \xi^{\prime}, \varepsilon\right)$ of the problems

$$
\begin{aligned}
A\left(\xi^{\prime}, D_{t}, \varepsilon\right) v(t) & =0, \quad t>0, \\
B_{k}\left(\xi^{\prime}, D_{t}, \varepsilon\right) v(0) & =\delta_{k j}, \quad k=1, \ldots, \nu, \\
v(t) & \rightarrow 0, \quad t \rightarrow+\infty .
\end{aligned}
$$

The set of these solutions is called the fundamental system of solutions of the problem (2.1). The description of the roots of (1.5) implies that the solutions of (2.12) exponentially decrease as $t \rightarrow \infty$. Therefore, for the fundamental system of solutions the integral norms are bounded:

$$
\begin{gathered}
\left\|D^{\ell} v_{j}\left(\cdot, \xi^{\prime}, \varepsilon\right)\right\|_{L_{2}\left(\mathbb{R}^{+}\right)}:=\left(\int_{0}^{+\infty}\left|D_{t}^{\ell} v_{j}\left(t, \xi^{\prime}, \varepsilon\right)\right|^{2} d t\right)^{1 / 2}<\infty, \\
j=1, \ldots, m, \quad \ell=0,1,2, \ldots
\end{gathered}
$$

One of the main results of the theory is the following theorem.

Theorem 2.6. Let Conditions $(\mathrm{A}),(\mathrm{B}),\left(\mathrm{C}_{1}\right),\left(\mathrm{C}_{2}\right),\left(\mathrm{C}_{3}\right)$ be satisfied. Then there exists $\varepsilon_{0}>0$ such that for $\varepsilon \leq \varepsilon_{0},\left|\xi^{\prime}\right|>0$ the integrals (2.13) are estimated as follows:

$$
\begin{aligned}
& \left\|D^{\ell} v_{j}\left(\cdot, \xi^{\prime}, \varepsilon\right)\right\|_{L_{2}\left(\mathbb{R}^{+}\right)} \leq \mathrm{const}\left|\xi^{\prime}\right|^{\ell-\beta_{j}-1 / 2} \\
& \quad \times \begin{cases}\left(1+\varepsilon\left|\xi^{\prime}\right|\right)^{\beta_{j}-b_{j}}, & j \leq \mu, \ell \leq \beta_{\mu+1} \\
\left(\varepsilon\left|\xi^{\prime}\right|\right)^{\beta_{\mu+1}-\ell+1 / 2}\left(1+\varepsilon\left|\xi^{\prime}\right|\right)^{\ell-\beta_{\mu+1}+\beta_{j}-b_{j}-1 / 2}, & j \leq \mu, \ell>\beta_{\mu+1} \\
\left(\varepsilon\left|\xi^{\prime}\right|\right)^{\beta_{j}-\beta_{\mu}}\left(1+\varepsilon\left|\xi^{\prime}\right|\right)^{-b_{j}+\beta_{\mu}}, & j>\mu, \ell \leq \beta_{\mu+1} \\
\left(\varepsilon\left|\xi^{\prime}\right|\right)^{\beta_{j}-\ell+1 / 2}\left(1+\varepsilon\left|\xi^{\prime}\right|\right)^{\ell-b_{j}-1 / 2}, & j>\mu, \ell>\beta_{\mu+1} .\end{cases}
\end{aligned}
$$


Estimates (2.14) depend on two parameters, $\varepsilon$ and $\left|\xi^{\prime}\right|$. In fact, one can easily reduce the proof to the case of one parameter.

Indeed, by homogeneity,

$$
A\left(\xi^{\prime}, D_{t}, \varepsilon\right) u(r t)=\left(A\left(\xi^{\prime}, r D_{t}, \varepsilon\right) u\right)(r t)=r^{2 \mu}\left(A\left(\xi^{\prime} / r, D_{t}, r \varepsilon\right) u\right)(r t) .
$$

Similarly,

$$
B_{k}\left(\xi^{\prime}, D_{t}, \varepsilon\right) u(r t)=r^{\beta_{k}}\left(B_{k}\left(\xi^{\prime} / r, D_{t}, r \varepsilon\right) u\right)(r t), \quad k=1, \ldots, m .
$$

Therefore the functions $v_{j}\left(t, \xi^{\prime}, \varepsilon\right)$ and $v_{j}\left(r t, \xi^{\prime} / r, r \varepsilon\right)$ satisfy equation (2.12) simultaneously. The uniqueness of the solution of this problem implies that

$$
v_{j}\left(t, \xi^{\prime}, \varepsilon\right)=r^{-\beta_{j}} v_{j}\left(r t, \xi^{\prime} / r, r \varepsilon\right) ;
$$

hence

$$
\left\|D^{\ell} v_{j}\left(\cdot, \xi^{\prime}, \varepsilon\right)\right\|_{L_{2}\left(\mathbb{R}^{+}\right)}=r^{\ell-\beta_{j}-1 / 2}\left\|D^{\ell} v_{j}\left(\cdot, \xi^{\prime} / r, r \varepsilon\right)\right\|_{L_{2}\left(\mathbb{R}^{+}\right)} .
$$

Setting $r=\left|\xi^{\prime}\right|$ and $\omega=\xi^{\prime} /\left|\xi^{\prime}\right|$ we obtain

$$
\left\|D^{\ell} v_{j}\left(\cdot, \xi^{\prime}, \varepsilon\right)\right\|_{L_{2}\left(\mathbb{R}^{+}\right)}=\left|\xi^{\prime}\right|^{\ell-\beta_{j}-1 / 2}\left\|D^{\ell} v_{j}\left(\cdot, \omega,\left|\xi^{\prime}\right| \varepsilon\right)\right\|_{L_{2}\left(\mathbb{R}^{+}\right)} .
$$

Therefore, (2.14) is an immediate consequence of the following estimate:

$$
\begin{aligned}
& \left\|D^{\ell} v_{j}(\cdot, \omega, \varepsilon)\right\|_{L_{2}\left(\mathbb{R}^{+}\right)} \leq \operatorname{const} \begin{cases}(1+\varepsilon)^{\beta_{j}-b_{j}}, & j \leq \mu, \ell \leq \beta_{\mu+1} ; \\
\varepsilon^{\beta_{\mu+1}-\ell+1 / 2}(1+\varepsilon)^{\ell-\beta_{\mu+1}+\beta_{j}-b_{j}-1 / 2}, & j \leq \mu, \ell>\beta_{\mu+1} ; \\
\varepsilon^{\beta_{j}-\beta_{\mu}}(1+\varepsilon)^{-b_{j}+\beta_{\mu}}, & j>\mu, \ell \leq \beta_{\mu+1} ; \\
\varepsilon^{\beta_{j}-\ell+1 / 2}(1+\varepsilon)^{\ell-b_{j}-1 / 2}, & j>\mu, \ell>\beta_{\mu+1} .\end{cases}
\end{aligned}
$$

In proving (2.16) we cannot restrict ourselves to the case of small $\varepsilon$ and should prove it for all $0<\varepsilon<\infty$. As was mentioned earlier (see (2.13)), for a fixed $\varepsilon$ the required estimate follows from Conditions (A) and (B). Therefore, inequality (2.16) should be proved separately for $\varepsilon \rightarrow 0$ and $\varepsilon \rightarrow \infty$. We start with the second case, which is simpler.

2.4. The proof of (2.16) for large $\varepsilon$. For $\varepsilon \rightarrow \infty$ the estimate (2.16) becomes

$$
\left\|D^{\ell} v_{j}(\cdot, \omega, \varepsilon)\right\|_{L_{2}\left(\mathbb{R}^{+}\right)} \leq \text {const } \varepsilon^{\beta_{j}-b_{j}} .
$$

To prove this inequality, we note that dividing in (2.12) the equation by $\varepsilon^{2 m-2 \mu}$ and the boundary condition by $\varepsilon^{b_{k}-\beta_{k}}$, we obtain the problem

$$
\begin{aligned}
\tilde{A}\left(\omega, D_{t}, 1 / \varepsilon\right) v_{j}(t) & =0, \quad t>0, \\
\tilde{B}_{k}\left(\omega, D_{t}, 1 / \varepsilon\right) v_{j}(0) & =\delta_{k j} \varepsilon^{\beta_{k}-b_{k}}, \quad k=1, \ldots, m, \\
v_{j}(t) & \rightarrow 0, \quad t \rightarrow+\infty,
\end{aligned}
$$

where

$$
\begin{gathered}
\tilde{A}\left(\omega, D_{t}, 1 / \varepsilon\right)=A_{2 m}\left(\omega, D_{t}\right)+\sum_{j \geq 1}^{2 m-2 \mu} \varepsilon^{-j} A_{2 m-j}\left(\omega, D_{t}\right), \\
\tilde{B}_{k}\left(\xi^{\prime}, D_{t}, 1 / \varepsilon\right)=B_{b_{k}}\left(\xi^{\prime}, D_{t}\right)+\sum_{j \geq 1}^{b_{k}-\beta_{k}} \varepsilon^{-j} B_{b_{k}-j}\left(\xi^{\prime}, D_{t}\right) .
\end{gathered}
$$


The obvious substitution $v_{j}=\varepsilon^{\beta_{j}-b_{j}} w_{j}$ yields

$$
\begin{aligned}
\tilde{A}\left(\omega, D_{t}, 1 / \varepsilon\right) w_{j}(t) & =0, \quad t>0, \\
\tilde{B}_{k}\left(\omega, D_{t}, 1 / \varepsilon\right) w_{j}(0) & =\delta_{k j}, \quad k=1, \ldots, m, \\
v_{j}(t) & \rightarrow 0, \quad t \rightarrow+\infty .
\end{aligned}
$$

Explicit expressions for the operators $\tilde{A}$ and $\tilde{B}_{k}$ show that for these operators there are small perturbations of the operators $A_{2 m}$ and $B_{b_{k}}$. Using Condition $\left(\mathrm{C}_{1}\right)$ and Lemma 2.3. we can prove that

$$
\left\|D^{\ell} w_{j}(\cdot, \omega, \varepsilon)\right\|_{L_{2}\left(\mathbb{R}^{+}\right)} \leq C
$$

with a constant independent of $\varepsilon$ as $\varepsilon \rightarrow \infty$, which implies (2.17).

\subsection{The proof of (2.16) for small $\varepsilon$.}

2.5.1. In this case the estimate takes the following form:

$$
\left\|D^{\ell} v_{j}(\cdot, \omega, \varepsilon)\right\|_{L_{2}\left(\mathbb{R}^{+}\right)} \leq \mathrm{const} \begin{cases}1, & j \leq \mu, \ell \leq \beta_{\mu+1} \\ \varepsilon^{\beta_{\mu+1}-\ell+1 / 2}, & j \leq \mu, \ell>\beta_{\mu+1} \\ \varepsilon^{\beta_{j}-\beta_{\mu}}, & j \mu, \ell \leq \beta_{\mu+1} \\ \varepsilon^{\beta_{j}-\ell+1 / 2}, & j \mu, \ell>\beta_{\mu+1} .\end{cases}
$$

Before proving this estimate, we present some auxiliary results.

2.5.2. Denote by $\tau_{1}^{+}(\omega), \ldots, \tau_{\mu}^{+}(\omega)$ the roots of the equation $A_{2 \mu}(\omega, \tau)=0$ in the halfplane $\mathbb{C}_{+}$. From the compactness of the sphere $\{\omega,|\omega|=1\}$ we see that there exists a contour $\gamma_{1} \in C_{+}$that lies at a positive distance from the real line and encircles all these roots at $|\omega|=1$.

According to Condition $\left(\mathrm{C}_{2}\right)$ and Lemma 2.1 there exist polynomials $N_{\ell}(\omega, \tau), \ell=$ $1, \ldots, \mu$ such that

$$
\frac{1}{2 \pi i} \int_{\gamma_{1}} \frac{B_{k}(\omega, \tau, 0) N_{\ell}(\tau, \omega)}{A_{2 \mu}^{+}(\omega, \tau)} d \tau=\delta_{k \ell}, \quad k, \ell=1, \ldots, \mu .
$$

(Recall that $B_{k}(\omega, \tau, 0)=B_{\beta_{k}}(\omega, \tau)$.)

Similarly, denote by $\tau_{\mu+1}^{+}, \ldots, \tau_{m}^{+}$the roots of the equation $Q(\tau)=0$ with positive imaginary parts and let the contour $\gamma_{2} \in C_{+}$encircle all these roots. According to Condition $\left(\mathrm{C}_{3}\right)$ and Lemma 2.1, there exist polynomials $N_{\ell}(\tau), \ell=\mu+1, \ldots, m$, such that

$$
\frac{1}{2 \pi i} \int_{\gamma_{2}} \frac{B_{k}(0, \tau, 1) N_{\ell}(\tau)}{Q^{+}(\tau)} d \tau=\delta_{k \ell}, \quad k, \ell=\mu+1, \ldots, m
$$

2.5.3. Denote by $\tau_{1}^{+}(\omega, \varepsilon), \ldots, \tau_{m}^{+}(\omega, \varepsilon)$ the roots of the equation $A(\omega, \tau, \varepsilon)=0$ in $\mathbb{C}_{+}$. According to Proposition 1.7, these roots split into two sets $S^{1+}(\omega, \varepsilon)$ and $S^{2+}(\omega, \varepsilon)$, and the above contour $\gamma_{1} \in C_{+}$encircles the set $S^{1+}(\omega, \varepsilon)$ when $|\omega|=1$ and $\varepsilon \leq \varepsilon_{0}$ is sufficiently small. Similarly, the contour $\gamma_{2} \in C_{+}$encircles the set $\varepsilon S^{2+}(\omega, \varepsilon)$ for each $|\omega|=1$ and sufficiently small $\varepsilon \leq \varepsilon_{0}$.

According to this splitting of the roots, we factor the symbol $A(\omega, \tau, \varepsilon)$ (see 1.4):

$$
A(\omega, \tau, \varepsilon)=A^{1+}(\omega, \tau, \varepsilon) A^{2+}(\omega, \tau, \varepsilon) A^{-}(\omega, \tau, \varepsilon) .
$$


Condition $\left(\mathrm{C}_{2}\right)$ and an analog of Lemma 2.3 for (2.8) imply that the following problem on the half-line is uniquely solvable for sufficiently small $\varepsilon<\varepsilon_{0}$ :

$$
\begin{aligned}
A\left(\omega, D_{t}, \varepsilon\right) v(t) & =0, \quad t>0, \\
B_{j}\left(\omega, D_{t}, \varepsilon\right) v(0) & =g_{j} \quad j=1, \ldots, \mu, \\
v(t) & \in \mathfrak{M}_{\gamma_{1}} .
\end{aligned}
$$

Moreover, there exist polynomials $N_{\ell}(\omega, \tau, \varepsilon), \ell=1, \ldots, \mu$, such that

$$
\frac{1}{2 \pi i} \int_{\gamma_{1}} \frac{B_{k}(\omega, \tau, \varepsilon) N_{\ell}(\tau, \omega, \varepsilon)}{A^{1+}(\tau, \omega, \varepsilon)} d \tau=\delta_{k \ell}, \quad k, \ell=1, \ldots, \mu
$$

It is useful to note that

$$
N_{\ell}(\tau, \omega, \varepsilon) \rightarrow N_{\ell}(\tau, \omega), \quad \varepsilon \rightarrow 0,
$$

and as $\varepsilon \rightarrow 0$, equalities (2.23) become equalities (2.20).

2.5.4. According to Condition $\left(\mathrm{C}_{3}\right)$ and an analog of Lemma 2.3 the following problem on the half-line is uniquely solvable for sufficiently small $\varepsilon<\varepsilon_{0}$ :

$$
\begin{aligned}
A\left(\varepsilon \omega, D_{t}, 1\right) v(t) & =0, \quad t>0, \\
B_{j}\left(\varepsilon \omega, D_{t}, 1\right) v(0) & =g_{j}, \quad j=1, \ldots, \mu, \\
v(t) & \in \mathfrak{M}_{\gamma_{2}} .
\end{aligned}
$$

Moreover, there exist polynomials $N_{\ell}(\omega, \tau, \varepsilon), \ell=\mu+1, \ldots, m$, such that

$$
\frac{1}{2 \pi i} \int_{\gamma_{2}} \frac{B_{k}(\varepsilon \omega, \tau, 1) N_{\ell}(\tau, \omega, \varepsilon)}{A^{2+}(\tau, \varepsilon \omega, 1)} d \tau=\delta_{k \ell}, \quad k, \ell=\mu+1, \ldots, m
$$

It is useful to note that

$$
N_{\ell}(\tau, \omega, \varepsilon) \rightarrow N_{\ell}(\tau), \quad \varepsilon \rightarrow 0, \quad \ell>\mu
$$

as $\varepsilon \rightarrow 0$, equalities (2.25) become equalities (2.21).

Let us note that (see (1.23))

$$
A^{2}\left(\omega, \frac{\tau}{\varepsilon}, \varepsilon\right)=A^{2}(\varepsilon \omega, \tau, 1), \quad B_{j}\left(\omega, \frac{\tau}{\varepsilon}, \varepsilon\right)=\varepsilon^{-\beta_{j}} B_{j}(\varepsilon \omega, \tau, 1) .
$$

Using these relations, we can rewrite (2.25) in the form

$$
\frac{1}{2 \pi i} \int_{\gamma_{2}} \frac{B_{k}\left(\omega, \frac{\tau}{\varepsilon}, \varepsilon\right) N_{\ell}(\tau, \omega, \varepsilon)}{A_{2}^{+}\left(\frac{\tau}{\varepsilon}, \omega, \varepsilon\right)} d \tau=\varepsilon^{-\beta_{k}} \delta_{k \ell}, \quad k, \ell=\mu+1, \ldots, m .
$$

2.5.5. Let us return to the proof of the estimate (2.19). Since at $|\omega|=1$ all roots of the equation (1.5) are encircled by the contours $\gamma_{1}$ and $\gamma_{2} / \varepsilon$, the space of functions on the half-line satisfying the homogeneous equation (2.12) is the direct sum of the subspaces $\mathfrak{M}_{\gamma_{1}}$ and $\mathfrak{M}_{\gamma_{2} / \varepsilon}$. We have the following result.

Lemma 2.7. The solution $v_{j}(t, \omega, \varepsilon)$ to the problem (2.12) can be represented in the form

$$
v_{j}(t, \omega, \varepsilon)=\frac{1}{2 \pi i} \int_{\gamma_{1}} \frac{M_{j}^{(1)}(\tau, \omega, \varepsilon)}{A^{1+}(\tau, \omega, \varepsilon)} e^{i t \tau} d \tau+\frac{1}{2 \pi i} \int_{\gamma_{2}} \frac{M_{j}^{(2)}(\tau, \omega, \varepsilon)}{A^{2+}(\tau, \varepsilon \omega, 1)} e^{i t \frac{\tau}{\varepsilon}} d \tau,
$$

where the functions $M_{j}^{(1)}$ and $M_{j}^{(2)}$ can be estimated as follows:

$$
\begin{aligned}
& \left|M_{j}^{(1)}(\tau, \omega, \varepsilon)\right| \leq \begin{cases}C, & j \leq \mu, \\
C \varepsilon^{\beta_{j}-\beta_{\mu}}, & j>\mu,\end{cases} \\
& \left|M_{j}^{(2)}(\tau, \omega, \varepsilon)\right| \leq \begin{cases}C \varepsilon^{\beta_{\mu+1}}, & j \leq \mu, \\
C \varepsilon^{\beta_{j}}, & j>\mu .\end{cases}
\end{aligned}
$$


These estimates and the representation (2.27) imply that

$$
\left\|\left(D_{t}^{\ell} v_{j}\right)(\cdot, \omega, \varepsilon)\right\|_{L_{2}\left(\mathbb{R}_{+}\right)} \leq \begin{cases}O(1)+O\left(\varepsilon^{\beta_{\mu+1}-\ell+\frac{1}{2}}\right), & j \leq \mu, \\ O\left(\varepsilon^{\beta_{j}-\beta_{\mu}}\right)+O\left(\varepsilon^{\beta_{j}-\ell+\frac{1}{2}}\right), & j>\mu .\end{cases}
$$

Inequalities (2.19) and, therefore, Theorem 2.6 follow immediately from these estimates.

Proof of Lemma 2.7. We use the Vishik-Lyusternik method in the form suggested by Frank [2]. The proof below repeats the proof given in [14 for the case of boundary conditions independent of the parameter.

Let $w(t, \omega, \varepsilon)$ be a solution to the problem (2.12) with $\delta_{j k}$ replaced by $\phi=\left(\phi_{1}, \ldots, \phi_{m}\right)$ $\in \mathbb{C}^{m}$. We look for the solution in the form

$$
\begin{aligned}
w(t, \omega, \varepsilon) & =\sum_{\ell=1}^{\mu} \psi_{\ell}(\omega, \varepsilon) \frac{1}{2 \pi i} \int_{\gamma-1} \frac{N_{\ell}(\tau, \omega, \varepsilon)}{A^{1+}(\tau, \omega, \varepsilon)} e^{i t \tau} d \tau \\
& +\sum_{\ell=\mu+1}^{m} \psi_{\ell}(\omega, \varepsilon) \frac{1}{2 \pi i} \int_{\gamma_{2}} \frac{N_{\ell}(\tau, \omega, \varepsilon)}{A^{2+}(\tau, \varepsilon \omega, 1)} e^{i t \frac{\tau}{\varepsilon}} d \tau
\end{aligned}
$$

with the functionals $\psi_{k}$ to be determined. The functions $N_{\ell}(\tau, \omega, \varepsilon)$ satisfy relations (2.23) for $\ell=1, \ldots, \mu$ and (2.25) for $\ell=\mu+1, \ldots, m$.

Applying to both parts of (2.31) the boundary operator $B_{k}\left(\omega, D_{t}, \varepsilon\right)$ and setting $t=0$, we obtain a system of linear equations for the unknown functions $\psi_{k}$ :

$$
\begin{gathered}
\psi_{k}(\omega, \varepsilon)+\varepsilon^{-\beta_{k}} \sum_{\ell=\mu+1}^{m} h_{k \ell}(\omega, \varepsilon) \psi_{\ell}(\omega, \varepsilon)=\phi_{k}, \quad k=1, \ldots, \mu, \\
\sum_{\ell=1}^{\mu} h_{k \ell}(\omega, \varepsilon) \psi_{\ell}(\omega, \varepsilon)+\varepsilon^{-\beta_{k}} \psi_{k}(\omega, \varepsilon)=\phi_{k}, \quad k=\mu+1, \ldots, m .
\end{gathered}
$$

Here we denote

$$
\begin{aligned}
h_{k \ell}(\omega, \varepsilon)=\frac{1}{2 \pi i} \int_{\gamma_{2}} \frac{B_{k}(\varepsilon \omega, \tau, 1) N_{\ell}(\tau, \omega, \varepsilon)}{A^{2+}(\tau, \varepsilon \omega, 1)} d \tau \\
(k=1, \ldots, \mu ; \ell=\mu+1, \ldots, m), \\
h_{k \ell}(\omega, \varepsilon)=\frac{1}{2 \pi i} \int_{\gamma_{1}} \frac{B_{k}(\tau, \omega, \varepsilon) N_{\ell}(\tau, \omega, \varepsilon)}{A^{1+}(\tau, \omega, \varepsilon)} d \tau \\
(k=\mu+1, \ldots, m ; \ell=1, \ldots, \mu) .
\end{aligned}
$$

Now we write $\psi=\left(\psi^{\prime}, \psi^{\prime \prime}\right)$, where $\psi^{\prime}$ consists of the first $\mu$ components of the vector $\psi$, and $\psi^{\prime \prime}$ of the remaining $m-\mu$ components. Similarly, $\phi=\left(\phi^{\prime}, \phi^{\prime \prime}\right)$. In this notation our system can be represented in the form

$$
\begin{aligned}
& \psi^{\prime}+\Delta_{1} H_{12} \psi^{\prime \prime}=\phi^{\prime}, \\
& H_{21} \psi^{\prime}+\Delta_{2} \psi^{\prime \prime}=\phi^{\prime \prime},
\end{aligned}
$$

where we use the notation

$$
\Delta_{1}:=\left(\begin{array}{ccc}
\varepsilon^{-\beta_{1}} & & \\
& \ddots & \\
& & \varepsilon^{-\beta_{\mu}}
\end{array}\right), \quad \Delta_{2}:=\left(\begin{array}{ccc}
\varepsilon^{-\beta_{\mu+1}} & & \\
& \ddots & \\
& & \varepsilon^{-\beta_{m}}
\end{array}\right)
$$

and

$$
H_{12}:=\left(h_{k \ell}\right)_{\substack{k=1, \ldots, \mu \\ \ell=\mu+1, \ldots, m}}^{k}, \quad H_{21}:=\left(h_{k \ell}\right)_{\substack{k=\mu+1, \ldots, m \\ \ell=1, \ldots, \mu}} .
$$


Multiplying the first equation from the left by $\Delta_{1} H_{12} \Delta_{2}^{-1}$ and subtracting the resulting equality from the second equation, we obtain

$$
\left(I-\Delta_{1} H_{12} \Delta_{2}^{-1} H_{21}\right) \psi^{\prime}=\phi^{\prime}-\Delta_{1} H_{12} \Delta_{2}^{-1} \phi^{\prime \prime} .
$$

Similarly,

$$
\left(I-\Delta_{2}^{-1} H_{21} \Delta_{1} H_{12}\right) \psi^{\prime \prime}=-\Delta_{2}^{-1} H_{21} \phi^{\prime}+\Delta_{2}^{-1} \phi^{\prime \prime} .
$$

The matrices in the parentheses in the left-hand sides of the above formulas differ from the identity matrix by matrices with entries not exceeding const $\varepsilon^{\beta_{\mu+1}-\beta_{\mu}}$. According to (2.4), $\beta_{\mu+1}-\beta_{\mu}>0$. Therefore, for small $\varepsilon \leq \varepsilon_{0}$ these matrices are invertible, and we denote the inverse matrices by $G_{1}$ and $G_{2}$ respectively. Then we have

$$
\begin{aligned}
\psi^{\prime} & =G_{1} \phi^{\prime}-G_{1} \Delta_{1} H_{12} \Delta_{2}^{-1} \phi^{\prime \prime}, \\
\psi^{\prime \prime} & =-G_{2} \Delta_{2}^{-1} H_{21} \phi^{\prime}+G_{2} \Delta_{2}^{-1} \phi^{\prime \prime} .
\end{aligned}
$$

Taking $\phi=e_{j}, 1 \leq j \leq \mu$, where $e_{j}$ is the $j$-th unit vector, we have

$$
\psi_{(j)}^{\prime}=G_{1} e_{j}, \quad \psi_{(j)}^{\prime \prime}=-G_{2} \Delta_{2}^{-1} H_{21} e_{j}, \quad j=1, \ldots, \mu .
$$

Similarly, for $j>\mu$ we have

$$
\psi_{(j)}^{\prime}=-G_{1} \Delta_{1} H_{12} \varepsilon^{\beta_{j}} e_{j}, \quad \psi_{(j)}^{\prime \prime}=G_{2} \varepsilon^{\beta_{j}} e_{j}, \quad j>\mu .
$$

Let us return to the representation (2.27). Denote by $\psi_{\ell(j)}^{\prime}, \ell=1, \ldots, \mu$, the components of the vector $\psi_{(j)}^{\prime}$ and by $\psi_{\ell(j)}^{\prime \prime}, \ell=\mu+1, \ldots, m$, the components of the vector $\psi_{(j)}^{\prime \prime}$. Then

$$
M_{j}^{(1)}=\sum_{\ell=1}^{\mu} \psi_{\ell(j)}^{\prime} N_{\ell}, \quad M_{j}^{(2)}=\sum_{\ell=\mu+1}^{m} \psi_{\ell(j)}^{\prime \prime} N_{\ell} .
$$

These formulas for $\psi_{(j)}^{\prime}$ and $\psi_{(j)}^{\prime \prime}$ imply that

$$
\psi_{\ell(j)}^{\prime}=O(1), \quad \psi_{\ell(j)}^{\prime \prime}=O\left(\varepsilon^{\beta_{\mu+1}}\right)
$$

for $j \leq \mu$ and

$$
\psi_{\ell(j)}^{\prime}=O\left(\varepsilon^{\beta_{j}-\beta_{\mu}}\right), \quad \psi_{\ell(j)}^{\prime \prime}=O\left(\varepsilon^{\beta_{j}}\right)
$$

for $j \leq \mu$. Therefore, we obtain the estimates (2.28) and (2.29).

2.6. Estimates of norms of fractional order for a fundamental system of solutions. Let $\ell=\ell_{0}+\ell_{1}$, where $\ell_{0}$ is a nonnegative integer and $0<\ell_{1}<1$. By the definition of the fractional order norm of a function $v(t)$ in one variable,

$$
\left\|D^{\ell} v(\cdot)\right\|_{L_{2}\left(\mathbb{R}^{+}\right)}^{2}:=\int_{0}^{+\infty} \int_{0}^{+\infty} \frac{\left|D_{t}^{\ell_{0}} v(t)-D_{t}^{\ell_{0}} v\left(t^{\prime}\right)\right|^{2}}{\left|t-t^{\prime}\right|^{1+2 \ell_{1}}} d t d t^{\prime}
$$

Theorem 2.8. In the hypotheses of Theorem 2.6 the estimates (2.14) hold for noninteger $\ell$.

To prove Theorem 2.8 we need a slight modification of the proof of Theorem 2.6.

1. Let us note that formula (2.15) holds for noninteger $\ell$. To prove this, let us note that

so that

$$
D^{\ell_{0}} v_{j}\left(t, \xi^{\prime}, \varepsilon\right)=\left|\xi^{\prime}\right|^{\ell_{0}-\beta_{j}} v_{j}\left(\left|\xi^{\prime}\right| t, \omega,\left|\xi^{\prime}\right| \varepsilon\right),
$$

$$
\begin{aligned}
& \left\|D^{\ell} v_{j}\left(\cdot, \xi^{\prime}, \varepsilon\right)\right\|_{L_{2}\left(\mathbb{R}^{+}\right)}^{2} \\
& \quad=\left|\xi^{\prime}\right|^{2 \ell_{0}-2 \beta_{j}} \int_{0}^{+\infty} \int_{0}^{+\infty} \frac{\left|D_{t}^{\ell_{0}} v_{j}\left(\left|\xi^{\prime}\right| t, \omega, \varepsilon\left|\xi^{\prime}\right|\right)-D_{t}^{\ell_{0}} v_{j}\left(\left|\xi^{\prime}\right| t^{\prime}, \omega, \varepsilon\left|\xi^{\prime}\right|\right)\right|}{\left|t-t^{\prime}\right|^{1+2 \ell_{1}}} d t d t^{\prime} .
\end{aligned}
$$

The substitution $\left(t, t^{\prime}\right) \rightarrow\left(\left|\xi^{\prime}\right| t,\left|\xi^{\prime}\right| t^{\prime}\right)$ yields (2.15). 
Therefore, for noninteger $\ell$ the proof of inequalities (2.14) can also be reduced to the proof of the estimate (2.16).

2. The proof of (2.16) for large $\varepsilon$ coincides with the arguments in 2.4 since the inequality

$$
\left\|D^{\ell} w_{j}(\cdot, \omega, \varepsilon)\right\|_{L_{2}\left(\mathbb{R}^{+}\right)} \leq C,
$$

with a constant independent of $\varepsilon$ for $\varepsilon \rightarrow \infty$, which was used in 2.4, can be easily extended to noninteger $\ell$.

3. To prove (2.16) for small $\varepsilon$ and noninteger $\ell$ we note that due to Lemma 2.7, it suffices to generalize (2.30) to noninteger values of $\ell$. To achieve this, we use the inequality

$$
\left\|D^{\ell} e^{i t \frac{\tau}{\varepsilon}}\right\|_{L_{2}\left(\mathbb{R}^{+}\right)} \leq C\left(\frac{\varepsilon}{\operatorname{Im} \tau}\right)^{\ell}
$$

2.7. An example of a small parameter elliptic problem (the Dirichlet problem). As an example of the operator we take an arbitrary operator of the form (0.3) such that at each inner point of the domain its inner symbol satisfies the condition of small parameter ellipticity, and at each boundary point it satisfies the condition of proper small parameter ellipticity. For the boundary conditions we take the Dirichlet conditions

$$
B_{j}\left(\xi^{\prime}, D_{t}, \varepsilon\right)=D_{t}^{j-1}, \quad j=1, \ldots, m .
$$

Let us verify that we obtain a small parameter elliptic problem, i.e., that all conditions of Definition 2.5 are satisfied. Condition (A) is satisfied by definition. To verify Condition (B), we use Lemma 2.1. Condition (ii) of this lemma means that the polynomials $B_{j}\left(\xi^{\prime}, \tau, \varepsilon\right)$ are linearly independent $\bmod A^{0+}\left(\tau, \xi^{\prime}, \varepsilon\right)$. For the polynomials $1, \tau, \ldots, \tau^{m-1}$ this is clearly true since the degree of $A^{0+}$ equals $m$.

One can similarly prove that the problems $A_{2 m}, B_{1}, \ldots, B_{m}$ and $A_{2 \mu}, B_{1}, \ldots, B_{\mu}$ are related by the Shapiro-Lopatinskii condition, i.e., Conditions $\left(\mathrm{C}_{1}\right)$ and $\left(\mathrm{C}_{2}\right)$ are satisfied.

Condition $\left(\mathrm{C}_{3}\right)$ means that the problem

$$
\begin{aligned}
Q\left(D_{t}\right) D_{t}^{\mu} v(t) & =0, \quad t>0, \\
D_{t}^{\mu-1+j} v(0) & =\phi_{j}, \quad j=1, \ldots, m-\mu, \\
v(t) & \rightarrow 0, \quad t \rightarrow+\infty
\end{aligned}
$$

is uniquely solvable. The substitution $z(t)=D_{t}^{\mu} v(t)$ reduces the problem (2.37) to the problem

$$
\begin{aligned}
Q\left(D_{t}\right) z(t) & =0, \quad t>0, \\
D_{t}^{j-1} z(0) & =\phi_{j}, \quad j=1, \ldots, m-\mu, \\
z(t) & \rightarrow 0, \quad t \rightarrow+\infty,
\end{aligned}
$$

which is uniquely solvable since the polynomial $Q(\tau)$ has no real roots and has $m-\mu$ roots in the upper half-plane. Now it remains to note that the differential equation $D_{t}^{\mu} v(t)=z(t)$ on the half-line $t>0$ has a unique solution tending to zero as $t \rightarrow \infty$ provided that $z(t) \rightarrow 0$ as $t \rightarrow \infty$.

\section{MAIN SPACES}

In this section we present necessary facts about function spaces with norms depending on a small parameter. In subsequent sections these norms will be used to prove a priori estimates. Rather than discussing the general case of a manifold with or without the boundary, we consider the cases of the entire space and of the half-space. 
3.1. The entire space. The space $H^{r, s}=H^{r, s}\left(\mathbb{R}^{n}\right), r \geq s$, is defined as the space of all elements of the Sobolev space $H^{r}$ with the norm

$$
\begin{aligned}
\|u\|_{r, s} & =\left\|\left(1+|D|^{2}\right)^{s / 2}\left(1+\varepsilon^{2}|D|^{2}\right)^{(r-s) / 2} u ; \mathbb{R}^{n}\right\| \\
& =\left(\int_{\mathbb{R}^{n}}\left(1+|\xi|^{2}\right)^{s}\left(1+\varepsilon^{2}|\xi|^{2}\right)^{(r-s)}|\hat{u}(\xi)|^{2} d \xi\right)^{1 / 2},
\end{aligned}
$$

where $\hat{u}(\xi)$ denotes the Fourier transform of the function $u(x)$.

Let us note that the inequality

$$
\left(1+|\xi|^{2}\right)^{s / 2}\left(1+\varepsilon^{2}|\xi|^{2}\right)^{(r-s) / 2} \leq C\left(1+|\xi|^{2}\right)^{s^{\prime} / 2}\left(1+\varepsilon^{2}|\xi|^{2}\right)^{\left(r^{\prime}-s^{\prime}\right) / 2}
$$

with $C$ independent of $\varepsilon$ holds if and only if $r \leq r^{\prime}, s \leq s^{\prime}$. In this case we say that there is the imbedding

$$
H^{r^{\prime}, s^{\prime}}\left(\mathbb{R}^{n}\right) \subset H^{r, s}\left(\mathbb{R}^{n}\right), \quad r \leq r^{\prime}, \quad s \leq s^{\prime} .
$$

Two functions $\Phi_{1}(\xi, \varepsilon)$ and $\Phi_{2}(\xi, \varepsilon)$ are said to be equivalent, denoted

$$
\Phi_{1}(\xi, \varepsilon) \approx \Phi_{2}(\xi, \varepsilon),
$$

if there exists a constant $C$ (independent of $\xi$ and $\varepsilon$ ) such that

$$
C^{-1} \Phi_{1}(\xi, \varepsilon) \leq \Phi_{2}(\xi, \varepsilon) \leq C \Phi_{1}(\xi, \varepsilon) .
$$

It is clear that if $\Phi_{1} \approx \Phi_{2}$, then $\Phi_{1}^{h} \approx \Phi_{2}^{h}$ for each $h$.

It is also clear that replacing the function $\left(1+|\xi|^{2}\right)^{s / 2}\left(1+\varepsilon^{2}|\xi|^{2}\right)^{(r-s) / 2}$ with an equivalent function, we obtain an equivalent norm in $H^{r, s}$.

In the case where $s$ is nonnegative and, say, $0 \leq \varepsilon \leq 1$, we have

$$
\left(1+|\xi|^{2}\right)^{s / 2}\left(1+\varepsilon^{2}|\xi|^{2}\right)^{(r-s) / 2} \approx 1+|\xi|^{s}\left(1+\varepsilon^{2}|\xi|^{2}\right)^{(r-s) / 2}
$$

and the norm (3.1) is equivalent to the norm

$$
\left\|u ; \mathbb{R}^{n}\right\|+\left\||D|^{s}\left(1+\varepsilon^{2}|D|^{2}\right)^{(r-s) / 2} u ; \mathbb{R}^{n}\right\| .
$$

For nonnegative integers $s$ and $r$ we indicate a norm that is equivalent to (3.2). This latter norm will play a prominent role in a priori estimates in the half-space. As before, we take $\xi=\left(\xi^{\prime}, \xi_{n}\right)$.

Lemma 3.1. For nonnegative integers $r$ and $s, 0 \leq s \leq r$, we have

$|\xi|^{2 s}\left(1+\varepsilon^{2}|\xi|^{2}\right)^{(r-s)} \approx \sum_{\ell=0}^{s}\left|\xi^{\prime}\right|^{2 s-2 \ell}\left(1+\varepsilon^{2}\left|\xi^{\prime}\right|^{2}\right)^{r-s} \xi_{n}^{2 \ell}+\sum_{\ell=s+1}^{r} \varepsilon^{2 \ell-2 s}\left(1+\varepsilon^{2}\left|\xi^{\prime}\right|^{2}\right)^{r-\ell} \xi_{n}^{2 \ell}$.

Introduce the notation

$$
\Xi_{\rho, \sigma}(\xi, \varepsilon)= \begin{cases}|\xi|^{\sigma}\left(1+\varepsilon^{2}|\xi|^{2}\right)^{(\rho-\sigma) / 2}, & \sigma \geq 0 \\ \varepsilon^{-\sigma}\left(1+\varepsilon^{2}|\xi|^{2}\right)^{\rho / 2}, & \sigma<0 .\end{cases}
$$

In this notation (3.3) can be rewritten as follows:

$$
\Xi_{r, s}(\xi, \varepsilon) \approx\left(\sum_{\ell=0}^{r} \Xi_{r-\ell, s-\ell}^{2}\left(\xi^{\prime}, \varepsilon\right) \xi_{n}^{2 \ell}\right)^{1 / 2} .
$$


Then for nonnegative integer numbers $r$ and $s$ the norm (3.2) is equivalent to the norm

$$
\begin{aligned}
\left|\left[u ; \mathbb{R}^{n}\right]\right|_{r, s} & :=\left\|u ; \mathbb{R}^{n}\right\|+\left(\sum_{\ell=0}^{r} \int_{-\infty}^{\infty}\left\|\Xi_{r-\ell, s-\ell}\left(D^{\prime}, \varepsilon\right) D_{n}^{\ell} u\left(\cdot, x_{n}\right) ; \mathbb{R}^{n-1}\right\|^{2} d x_{n}\right)^{1 / 2} \\
& =\left\|u ; \mathbb{R}^{n}\right\|+\left(\int_{\mathbb{R}^{n}} \sum_{\ell=0}^{r} \Xi_{r-\ell, s-\ell}^{2}\left(\xi^{\prime}, \varepsilon\right)\left|D_{n}^{\ell} \hat{u}^{\prime}\left(\xi^{\prime}, x_{n}\right)\right|^{2} d \xi^{\prime} d x_{n}\right)^{1 / 2}
\end{aligned}
$$

where $\hat{u}^{\prime}\left(\xi^{\prime}, x_{n}\right)$ denotes the partial Fourier transform of the function $u(x)$ in the variables $x^{\prime}$.

The proof of Lemma 3.1. Replacing $|\xi|^{2}$ with $\left|\xi^{\prime}\right|^{2}+\xi_{n}^{2}$ and expanding the function in the left-hand side of (3.3) in powers of $\xi_{n}^{2}$ using the binomial expansion, we obtain

$$
\sum_{\ell_{1}=0}^{s} \sum_{\ell_{2}=0}^{r-s}\left(\begin{array}{c}
s \\
\ell_{1}
\end{array}\right)\left(\begin{array}{c}
r-s \\
\ell_{2}
\end{array}\right)\left|\xi^{\prime}\right|^{2 s-2 \ell_{1}}\left(1+\varepsilon^{2}\left|\xi^{\prime}\right|^{2}\right)^{r-s-\ell_{2}} \varepsilon^{2 \ell_{2}} \xi_{n}^{2 \ell_{1}+2 \ell_{2}}
$$

Taking in (3.6) the terms corresponding to $\ell_{2}=0$ we obtain

$$
\sum_{\ell=0}^{s}\left(\begin{array}{l}
s \\
\ell
\end{array}\right)\left|\xi^{\prime}\right|^{2 s-2 \ell}\left(1+\varepsilon^{2}\left|\xi^{\prime}\right|^{2}\right)^{r-s} \xi_{n}^{2 \ell} .
$$

Taking in (3.6) the terms corresponding to $\ell_{1}=s$ we obtain

$$
\sum_{\ell=s}^{r}\left(\begin{array}{c}
r-s \\
\ell
\end{array}\right) \varepsilon^{2 \ell-2 s}\left(1+\varepsilon^{2}\left|\xi^{\prime}\right|^{2}\right)^{r-\ell} \xi_{n}^{2 \ell} .
$$

This shows that the right-hand side of (3.3) can be estimated by the left-hand side with the coefficient 1 .

Now we estimate the left-hand side from above by the right-hand side with some positive constant. We consider separately the cases of "large" and "small" values of $\left|\xi_{n}\right|$. For $\left|\xi_{n}\right| \leq\left|\xi^{\prime}\right|$ the function in the left-hand side of (3.3) can be estimated from above by

$$
2^{r}\left|\xi^{\prime}\right|^{2 s}\left(1+\varepsilon^{2}\left|\xi^{\prime}\right|^{2}\right)^{r-s},
$$

i.e., by the first summand in the right-hand side. For $\left|\xi_{n}\right|>\left|\xi^{\prime}\right|$ the left-hand side can be estimated from above by

$$
2^{r} \xi_{n}^{2 s}\left(1+\varepsilon^{2} \xi_{n}^{2}\right)^{r-s} \leq 2^{4 r-2 s}\left(\xi_{n}^{2 s}+\varepsilon^{2 r-2 s} \xi_{n}^{2 r}\right) .
$$

Now it remains to note that the sum of terms in (3.3) corresponding to $\ell=s$ and $\ell=r$ equals

$$
\left(1+\varepsilon^{2}\left|\xi^{\prime}\right|^{2}\right)^{r-s} \xi_{n}^{2 s}+\varepsilon^{2 r-2 s}\left|\xi_{n}\right|^{2 r}>\xi_{n}^{2 s}+\varepsilon^{2 r-2 s} \xi_{n}^{2 r} .
$$

3.2. The half-space. Following the general definition of a function space in a domain, we define $H^{r, s}\left(\mathbb{R}_{+}^{n}\right)$ as the quotient space $H^{r, s}\left(\mathbb{R}^{n}\right) / H^{r, s}\left(\mathbb{R}^{n}\right)_{-}$, where $H^{r, s}\left(\mathbb{R}^{n}\right)_{-}$is the subspace of $H^{r, s}\left(\mathbb{R}^{n}\right)$ consisting of the function (distributions) with support in the half-space $\mathbb{R}_{-}^{n}=\{(x, t), t \leq 0\}$. We endow $H^{r, s}\left(\mathbb{R}_{+}^{n}\right)$ with the natural quotient norm

$$
\left\|u ; \mathbb{R}_{+}^{n}\right\|_{r, s}:=\inf _{L u \in T(u)}\left\|L u ; \mathbb{R}^{n}\right\|_{r, s},
$$

where $T(u)$ is the coset of the function $u$ and $L u$ runs over all representatives of this coset. Let us note that different representatives of the same coset $T(u)$ differ from one another only at the points where $t<0$; therefore, for $r \geq 0$, elements of the quotient space can be identified with functions defined on the half-space, and the functions $L u$ are the extensions of $u$ to the entire space. 
Inclusions of the spaces $H^{r, s}\left(\mathbb{R}^{n}\right)$ induce the natural inclusions

$$
H^{r^{\prime}, s^{\prime}}\left(\mathbb{R}_{+}^{n}\right) \subset H^{r, s}\left(\mathbb{R}_{+}^{n}\right), \quad r \leq r^{\prime}, \quad s \leq s^{\prime} .
$$

Let us note that equivalent norms in $H^{r, s}\left(\mathbb{R}^{n}\right)$ induce equivalent norms in $H^{r, s}\left(\mathbb{R}_{+}^{n}\right)$. In particular, the norm (3.7) is equivalent to the norm

$$
\left\|u ; \mathbb{R}_{+}^{n}\right\|_{r, s}:=\inf _{L u \in T(u)}\left(\left\|L u ; \mathbb{R}^{n}\right\|+\left\||D|^{s}\left(1+\varepsilon^{2}|D|^{2}\right)^{(r-s) / 2} L u ; \mathbb{R}^{n}\right\|\right), \quad s \geq 0 .
$$

Indeed, the equivalence of norms in $\mathbb{R}^{n}$ implies the existence of a constant $C$ such that

$$
\left\|L u ; \mathbb{R}^{n}\right\|_{r, s} \leq C\left(\left\|L u ; \mathbb{R}^{n}\right\|+\left\||D|^{s}\left(1+\varepsilon^{2}|D|^{2}\right)^{(r-s) / 2} L u ; \mathbb{R}^{n}\right\|\right) .
$$

The left-hand side can be estimated from below by $\left\|u ; \mathbb{R}_{+}^{n}\right\|_{r, s}$. Taking the lower bound of the right-hand side over all extensions $L u$ we estimate the norm (3.7) in terms of (3.8). Similarly one proves the opposite estimate.

Proposition 3.2. For nonnegative integers $r$ and $s, 0 \leq s \leq r$, the norms (3.7) and (3.8) are equivalent to the norm

$$
\left|\left[u ; \mathbb{R}_{+}^{n}\right]\right|_{r, s}:=\left\|u ; \mathbb{R}_{+}^{n}\right\|+\left(\sum_{\ell=0}^{r} \int_{0}^{\infty}\left\|\Xi_{r-\ell, s-\ell}\left(D^{\prime}, \varepsilon\right) D_{n}^{\ell} u\left(\cdot, x_{n}\right) ; \mathbb{R}^{n-1}\right\|^{2} d x_{n}\right)^{1 / 2}
$$

Proof. It is clear that for an arbitrary extension $L u \in H^{r, s}\left(\mathbb{R}^{n}\right)$ we have

$$
\int_{0}^{\infty}\left\|\Xi_{r-\ell, s-\ell}\left(D^{\prime}, \varepsilon\right) D_{n}^{\ell} u\left(\cdot, x_{n}\right) ; \mathbb{R}^{n-1}\right\|^{2} d x_{n} \leq\left\|\Xi_{r-\ell, s-\ell}\left(D^{\prime}, \varepsilon\right) D_{n}^{\ell} L u ; \mathbb{R}^{n}\right\| .
$$

Using Lemma 3.1 and taking the lower bound over all extensions $L u$, we estimate the right-hand side of (3.9) in terms of the norm (3.8), hence in terms of the norm (3.7).

To prove the opposite inequality we use a special construction of a linear bounded extension operator

$$
E: H^{r, s}\left(\mathbb{R}_{+}^{n}\right) \rightarrow H^{r, s}\left(\mathbb{R}^{n}\right)
$$

According to the definition and Lemma 3.1, the norm (3.8) is estimated from above by $\left|\left[E u ; \mathbb{R}^{n}\right]\right|_{r, s}$. If the operator $E$ satisfies the conditions

$$
\int_{-\infty}^{+\infty}\left|D_{n}^{\ell} \widehat{E u}^{\prime}\left(\xi^{\prime}, x_{n}\right)\right|^{2} d x_{n} \leq \mathrm{const} \int_{0}^{\infty}\left|D_{n}^{\ell} \hat{u}^{\prime}\left(\xi^{\prime}, x_{n}\right)\right|^{2} d x_{n}
$$

we estimate the norm (3.8) in terms of the right-hand side of (3.9).

For the extension operator $E$ satisfying (3.10) we can take the Hestenes extension operator

$$
(E u)\left(x^{\prime}, x_{n}\right)= \begin{cases}u\left(x^{\prime}, x_{n}\right), & x_{n} \geq 0 \\ \sum_{k=0}^{N} a_{k} u\left(x^{\prime},-\frac{1}{k} x_{n}\right), & x_{n}<0\end{cases}
$$

where $N$ is sufficiently large and the numbers $a_{k}$ satisfy the conditions

$$
\sum_{k=0}^{N} a_{k}\left(-\frac{1}{k}\right)^{j}=1, \quad j=0,1, \ldots, N
$$

Let us make an important remark. In defining the norm in $H^{r, s}\left(\mathbb{R}_{+}^{n}\right)$ it is convenient to replace the powers of the differential operators $1+|D|^{2}$ and $1+\varepsilon^{2}|D|^{2}$ by the powers of the pseudodifferential operators $D_{n}-i \sqrt{1+\left|D^{\prime}\right|^{2}}$ and $\varepsilon D_{n}-i \sqrt{1+\varepsilon^{2}\left|D^{\prime}\right|^{2}}$. Then the norm (3.7) becomes equivalent to the norm

$$
\left\|R\left(D_{n}-i \sqrt{1+\left|D^{\prime}\right|^{2}}\right)^{s}\left(\varepsilon D_{n}-i \sqrt{1+\varepsilon^{2}\left|D^{\prime}\right|^{2}}\right)^{r-s} L u ; \mathbb{R}_{+}^{n}\right\|,
$$


where $R$ is the operator of restriction from $\mathbb{R}^{n}$ to $\mathbb{R}_{+}^{n}$. Indeed, since the symbol

$$
\left(\xi_{n}-i \sqrt{1+\left|\xi^{\prime}\right|^{2}}\right)^{s}\left(\varepsilon \xi_{n}-i \sqrt{1+\varepsilon^{2}\left|\xi^{\prime}\right|^{2}}\right)^{r-s}
$$

is holomorphic at $\operatorname{Im} \xi_{n}<0$, the corresponding pseudodifferential operator transforms the functions supported on the half-space $x_{n}<0$ to the functions of the same type. Therefore, the norm (3.11) does not depend on the choice of the extension $L u$.

3.3. Traces of functions from the spaces $H^{r, s}\left(\mathbb{R}^{n}\right)$ and $H^{r, s}\left(\mathbb{R}_{+}^{n}\right)$. Since for $\varepsilon>0$ the space $H^{r, s}\left(\mathbb{R}^{n}\right)$ is a subset of the Sobolev space $H^{r}\left(\mathbb{R}^{n}\right)$, for $r>\ell+1 / 2$ one can define the trace operator $\mathcal{T}_{\ell}: u(x) \rightarrow D_{n}^{\ell} u\left(x^{\prime}, 0\right)$. We indicate the norms in which the trace operators are uniformly bounded as $\varepsilon \rightarrow 0$.

Using the notation (3.4), we define the space $\mathcal{H}^{\rho, \sigma}\left(\mathbb{R}^{n-1}\right)$ of functions $f\left(x^{\prime}\right)$ with the norm

$$
\left\|f, \mathcal{H}^{\rho, \sigma}\left(\mathbb{R}^{n-1}\right)\right\|= \begin{cases}\left\|f, \mathbb{R}^{n-1}\right\|+\left\|\Xi_{\rho, \sigma}\left(D^{\prime}, \varepsilon\right) f, \mathbb{R}^{n-1}\right\|, & \sigma \geq 0 \\ \left\|\Xi_{\rho, \sigma}\left(D^{\prime}, \varepsilon\right) f, \mathbb{R}^{n-1}\right\|, & \sigma<0 .\end{cases}
$$

This definition implies that $\mathcal{H}^{\rho, \sigma}\left(\mathbb{R}^{n-1}\right)$ coincides with $H^{\rho, \sigma}\left(\mathbb{R}^{n-1}\right)$ for $\sigma \geq 0$.

Proposition 3.3. For $r>\ell+1 / 2, s \geq 0$, and $s \neq \ell+1 / 2$ we have

$$
\left\|\left(D_{n}^{\ell} f\right)(\cdot, 0) ; \mathcal{H}^{r-\ell-1 / 2, s-\ell-1 / 2}\left(\mathbb{R}^{n-1}\right)\right\| \leq \text { const }\left\|f ; \mathbb{R}^{n}\right\|_{r, s}
$$

with a constant independent of $\varepsilon$.

Proof. Inequality (3.13) follows from a more general result presented in [18, Theorem 6.1]. According to this result, for a positive function $\chi(\xi)$ satisfying the condition $\chi(\xi) / \chi(\eta) \leq$ const $(1+|\xi-\eta|)^{M}$ with some positive $M$ we have

$$
\left|\left(\chi_{\ell}^{\prime}\left(D^{\prime}\right) D_{n}^{\ell} f\right)(\cdot, 0) ; \mathbb{R}^{n-1}\right| \leq \mathrm{const}\left\|\chi(D) f ; \mathbb{R}^{n}\right\|,
$$

where

$$
\chi_{\ell}^{\prime}\left(\xi^{\prime}\right)=\left(\int_{-\infty}^{-\infty} \xi_{n}^{2 \ell} \chi^{-2}\left(\xi^{\prime}, \xi_{n}\right) d \xi_{n}\right)^{-1 / 2}
$$

Now for $\chi(\xi)$ in the right-hand side of (3.15) we take the function

$$
\left(1+|\xi|^{2}\right)^{s / 2}\left(1+\varepsilon^{2}|\xi|^{2}\right)^{(r-s) / 2} .
$$

For $s>\ell+1 / 2$ let us take $\xi_{n}=\left(1+\left|\xi^{\prime}\right|^{2}\right)^{1 / 2} t$. Then in the right-hand side of (3.15) we obtain

$$
\left(1+\left|\xi^{\prime}\right|^{2}\right)^{-s+\ell+1 / 2}\left(1+\varepsilon^{2}\left|\xi^{\prime}\right|^{2}\right)^{-r+s} \int_{-\infty}^{\infty} t^{2 \ell}\left(1+t^{2}\right)^{-s}\left(1+\frac{\varepsilon^{2}+\varepsilon^{2}\left|\xi^{\prime}\right|^{2}}{1+\varepsilon^{2}\left|\xi^{\prime}\right|^{2}} t^{2}\right)^{-r+s} d t
$$

For every $\varepsilon$ the integrand can be estimated by $t^{2 \ell}\left(1+t^{2}\right)^{-s}$, and, say, for $\varepsilon \leq 1$ it can be estimated from below by $t^{2 \ell}\left(1+t^{2}\right)^{-r}$. Therefore, the integral can be estimated from above and from below by constants independent of $\varepsilon$ and we can take

$$
\chi_{\ell}^{\prime}\left(\xi^{\prime}\right):=\left(1+\left|\xi^{\prime}\right|^{2}\right)^{-s+\ell+1 / 2}\left(1+\varepsilon^{2}\left|\xi^{\prime}\right|^{2}\right)^{-r+s} \approx\left(1+\Xi_{r-\ell-1 / 2, s-\ell-1 / 2}^{2}\left(\xi^{\prime}\right)\right)^{1 / 2} .
$$

Now consider the case $0 \leq s<\ell+1 / 2$. In this case we take $\xi_{n}=\varepsilon^{-1}\left(1+\varepsilon^{2}\left|\xi^{\prime}\right|^{2}\right)^{1 / 2} t$. Then in the right-hand side of (3.15) we obtain

$$
\varepsilon^{2 s-2 \ell-1}\left(1+\varepsilon^{2}\left|\xi^{\prime}\right|^{2}\right)^{-r+2 \ell+1} \int_{-\infty}^{\infty} t^{2 \ell}\left(\frac{\varepsilon^{2}+\varepsilon^{2}\left|\xi^{\prime}\right|^{2}}{1+\varepsilon^{2}\left|\xi^{\prime}\right|^{2}}+t^{2}\right)^{-s}\left(1+t^{2}\right)^{-r+s} d t .
$$

For every $\varepsilon$ the integrand can be estimated from above by the absolutely integrable function $t^{2 \ell-2 s}\left(1+t^{2}\right)^{-r+s}$ and for, say, $0 \leq s<\ell+1 / 2$ we can take

$$
\chi_{\ell}^{\prime}\left(\xi^{\prime}\right)=\Xi_{r-\ell-1 / 2, s-\ell-1 / 2}\left(\xi^{\prime}, \varepsilon\right) \text {. }
$$


The definition (3.7) of the space $H^{r, s}\left(\mathbb{R}_{+}^{n}\right)$ and inequality (3.13) imply that for $r>$ $\ell+1 / 2$ and $s \neq \ell+1 / 2$ the function $f(x) \in H^{r, s}\left(\mathbb{R}_{+}^{n}\right)$ has the trace

$$
\left(D_{n}^{\ell} f\right)(\cdot, 0) \in \mathcal{H}^{r-\ell-1 / 2, s-\ell-1 / 2}\left(\mathbb{R}^{n-1}\right)
$$

and

$$
\left\|\left(D_{n}^{\ell} f\right)(\cdot, 0) ; \mathcal{H}^{r-\ell-1 / 2, s-\ell-1 / 2}\left(\mathbb{R}^{n-1}\right)\right\| \leq \text { const }\left\|f ; \mathbb{R}_{+}^{n}\right\|_{r, s}
$$

with a constant independent of $\varepsilon$.

3.4. The spaces $H^{r, s}\left(\mathbb{R}^{n}\right)$ and $H^{r, s}\left(\mathbb{R}_{+}^{n}\right)$ for negative integer $s$. Formally the norm (3.1) can be defined for negative integer numbers $s<0$ as well, and we can consider the spaces with such a norm. S. A. Nazarov [5] suggested defining the space $H^{r, s}\left(\mathbb{R}^{n}\right)$ for $s<0$ as a smaller set consisting of distributions for which the seminorm

$$
{ }^{\prime}\left\|u ; \mathbb{R}^{n}\right\|_{r, s}:=\left\||D|^{s}\left(1+\varepsilon^{2}|D|^{2}\right)^{(r-s) / 2} u ; \mathbb{R}^{n}\right\|=\left\||D|^{s} u ; \mathbb{R}^{n}\right\|_{r-s, 0}
$$

is finite. We have already used the seminorm (3.17) for $s \geq 0$ and have already noted in 3.1 that for $s \geq 0$ we have

$$
\left\|u ; \mathbb{R}^{n}\right\|_{r, s} \equiv\left\|u ; \mathbb{R}^{n}\right\|+{ }^{\prime}\left\|u ; \mathbb{R}^{n}\right\|_{r, s} .
$$

For $s<0$ it is clear that

$$
\left\|u ; \mathbb{R}^{n}\right\|_{r, s} \leq^{\prime}\left\|u ; \mathbb{R}^{n}\right\|_{r, s} .
$$

The seminorm (3.17) determines the set of distributions $u$ such that the Fourier transform $\hat{u}(\xi)$ has a zero of order $-s$ at the point $\xi=0$.

The space $H^{r, s}\left(\mathbb{R}^{n}\right)$ for negative integers $s$ has the following description (see [5]).

Proposition 3.4. Let $s$ be a negative integer. A distribution $u$ is in $H^{r, s}\left(\mathbb{R}^{n}\right)$ if and only if it can be represented in the form

$$
u=\sum_{|\alpha|=-s} D^{\alpha} u_{\alpha}, \quad u_{\alpha} \in H^{r-s, 0}\left(\mathbb{R}^{n}\right) .
$$

With such a representation, the norm (3.17) is equivalent to the norm

$$
{ }^{\prime}\left\|u ; \mathbb{R}^{n}\right\|_{r, s}=\inf \sum_{\alpha}\left\|u_{\alpha} ; \mathbb{R}^{n}\right\|_{r-s, 0},
$$

where the lower bound is taken over all representations (3.18).

Proof. If a distribution $u$ can be represented in the form (3.18), then it lies in $H^{r, s}$ and

$$
{ }^{\prime}\left\|u ; \mathbb{R}^{n}\right\|_{r, s} \leq \sum_{|\alpha|=-s}\left\||D|^{s} D^{\alpha} u_{\alpha} ; \mathbb{R}^{n}\right\|_{r-s, 0} \leq \sum_{\alpha}\left\|u_{\alpha} ; \mathbb{R}^{n}\right\|_{r-s, 0},
$$

which implies the inequality

$$
{ }^{\prime}\left\|u ; \mathbb{R}^{n}\right\|_{r, s} \leq{ }^{\prime \prime}\left\|u ; \mathbb{R}^{n}\right\|_{r, s} .
$$

On the other hand, according to the original definition, each representation $u \in H^{r, s}$ can be represented in the form $u=|D|^{-s} u_{0}$, where $u_{0} \in H^{r-s, 0}$. Take the binomial expansion of $|D|^{-s}$,

$$
|D|^{-s}=\left(\sum_{j=1}^{n} \frac{D_{j}}{|D|} D_{j}\right)^{-s}=\sum_{|\alpha|=-s} c_{\alpha}\left(\frac{D_{1}}{|D|}, \ldots, \frac{D_{n}}{|D|}\right) D^{\alpha},
$$

where $c_{\alpha}\left(\omega_{1}, \ldots, \omega_{n}\right)$ are homogeneous functions of degree zero. Setting

$$
u_{\alpha}=c_{\alpha}\left(\frac{D_{1}}{|D|}, \ldots, \frac{D_{n}}{|D|}\right) u_{0}
$$


we arrive at the representation (3.18); moreover,

$$
{ }^{\prime \prime}\left\|u ; \mathbb{R}^{n}\right\|_{r, s} \leq \sum_{\alpha}\left\|u_{\alpha} ; \mathbb{R}^{n}\right\|_{r-s, 0} \leq \text { const }\left\|u_{0}\right\|_{r-s, 0}=\text { const }^{\prime}\left\|u ; \mathbb{R}^{n}\right\|_{r, s} .
$$

The space $H^{r, s}\left(\mathbb{R}_{+}^{n}\right), s<0$, is naturally defined as the quotient space of the space $H^{r, s}\left(\mathbb{R}^{n}\right)$ by the subspace consisting of distributions supported on the half-space $x_{n}<0$. We endow this space with the natural quotient norm. According to (3.11) for this norm we can take

$$
{ }^{\prime}\left\|u ; \mathbb{R}_{+}^{n}\right\|_{r, s}=\left\|R\left(D_{n}-i\left|D^{\prime}\right|\right)^{s}\left(\varepsilon D_{n}-i \sqrt{1+\varepsilon^{2}\left|D^{\prime}\right|^{2}}\right)^{r-s} L u ; \mathbb{R}^{n}\right\| .
$$

For the space $H^{r, s}\left(\mathbb{R}_{+}^{n}\right)$ we have a direct analog of Proposition 3.4.

Proposition 3.5. Let $s$ be a negative integer number. A distribution $u$ is in $H^{r, s}\left(\mathbb{R}_{+}^{n}\right)$ if and only if it can be represented in the form

$$
u=\sum_{|\alpha|=-s} D^{\alpha} u_{\alpha}, \quad u_{\alpha} \in H^{r-s, 0}\left(\mathbb{R}_{+}^{n}\right) .
$$

With such a representation, the norm (3.20) is equivalent to the norm

$$
{ }^{\prime \prime}\left\|u ; \mathbb{R}_{+}^{n}\right\|_{r, s}=\inf \sum_{\alpha}\left\|u_{\alpha} ; \mathbb{R}_{+}^{n}\right\|_{r-s, 0},
$$

where the lower bound is taken over all representations (3.21).

The proof repeats the proof of Proposition 3.4. The inequality

$$
'\left\|u ; \mathbb{R}_{+}^{n}\right\|_{r, s} \leq{ }^{\prime \prime}\left\|u ; \mathbb{R}_{+}^{n}\right\|_{r, s}
$$

follows from the definition of the norm (3.20) and the boundedness of the operator $\left(D_{n}-i\left|D^{\prime}\right|\right)^{s} D^{\alpha},|\alpha|=-s$, in the space $H^{r-s, 0}\left(\mathbb{R}^{n}\right)$. As in the proof of Proposition 3.4, the proof of the opposite inequality is based on the binomial expansion of the operator $\left(D_{n}-i\left|D^{\prime}\right|\right)^{-s}$ :

$$
\left(D_{n}-i\left|D^{\prime}\right|\right)^{-s}=\left(D_{n}-i \sum_{j=1}^{n-1} \frac{D_{j}}{\left|D^{\prime}\right|} D_{j}\right)^{-s}=\sum_{|\alpha|=-s} c_{\alpha}\left(\frac{D_{1}}{\left|D^{\prime}\right|}, \ldots, \frac{D_{n-1}}{\left|D^{\prime}\right|}\right) D^{\alpha} .
$$

In conclusion, we consider the traces of the functions in $H^{r, s}\left(\mathbb{R}^{n}\right)$ and $H^{r, s}\left(\mathbb{R}_{+}^{n}\right)$ for $s<0$. The existence of $\left(D_{n}^{\ell} f\right)(\cdot, 0)$ is proved using the inequality $r>\ell+1 / 2$. For $s<0$ we modify the norm (3.13) by setting

$$
{ }^{\prime}\left\|f, \mathcal{H}^{\rho, \sigma}\left(\mathbb{R}^{n-1}\right)\right\|=\left\|\Xi_{\rho, \sigma}\left(D^{\prime}, \varepsilon\right) f, \mathbb{R}^{n-1}\right\| .
$$

This norm is defined for all $\sigma>0$. Proposition 3.3 remains true for negative $s$ if we replace (3.14) with

$$
'\left\|\left(D_{n}^{\ell} f\right)(\cdot, 0) ; \mathcal{H}^{r-\ell-1 / 2, s-\ell-1 / 2}\left(\mathbb{R}^{n-1}\right)\right\| \leq \text { const }^{\prime}\left\|f ; \mathbb{R}^{n}\right\|_{r, s} .
$$

\section{A PRIORI ESTIMATES IN THE HALF-SPACE}

4.1. The formulation of the problem. This section is devoted to a priori estimates in the half-space for solutions of the problem

$$
\begin{aligned}
A\left(D^{\prime}, D_{n}, \varepsilon\right) u(x) & =f(x), \quad x_{n}>0, \\
\left(B_{j}\left(D^{\prime}, D_{n}, \varepsilon\right) u\right)\left(x^{\prime}, 0\right) & =g_{j}\left(x^{\prime}\right), \quad j=1, \ldots, m .
\end{aligned}
$$


We will look for estimates in the spaces $H^{r, s}\left(\mathbb{R}_{+}^{n}\right)$ with $r>s \geq 0$. We will not be concerned with restrictions on $r$ from above and will assume that

$$
\begin{gathered}
r-s \geq 2 m-2 \mu, \quad r-s \geq b_{j}-\beta_{j}, \quad r>b_{j}+\frac{1}{2}, \\
j=1, \ldots, m .
\end{gathered}
$$

Later on, these conditions will not be mentioned explicitly. As for $s$, we will see that one can obtain the required estimates only for a relatively narrow range of this parameter.

Let us recall that $A(D, \varepsilon)$ is a quasihomogeneous operator of the form

$$
A(D, \varepsilon)=\sum_{\ell=0}^{2 m-2 \mu} \varepsilon^{2 m-2 \mu-\ell} A_{\ell}(D), \quad \text { ord } A_{\ell}(D)=2 m-\ell .
$$

Similarly, $B_{j}(D, \varepsilon)$ are quasihomogeneous operators of the form

$$
B_{j}(D, \varepsilon)=\sum_{\ell=0}^{b_{j}-\beta_{j}} \varepsilon^{b_{j}-\beta_{j}-\ell} B_{j \ell}(D), \quad \text { ord } B_{j \ell}(D)=b_{j}-\ell .
$$

The form of these operators implies the following inequalities:

$$
\begin{aligned}
& { }^{\prime}\left\|A(D, \varepsilon) u ; \mathbb{R}^{n}\right\|_{r-2 m, s-2 \mu} \leq \mathrm{const}^{\prime}\left\|u ; \mathbb{R}^{n}\right\|_{r, s}, \\
& { }^{\prime}\left\|B_{j}(D, \varepsilon) u ; \mathbb{R}^{n}\right\|_{r-b_{j}, s-\beta_{j}} \leq \mathrm{const}^{\prime}\left\|u ; \mathbb{R}^{n}\right\|_{r, s} .
\end{aligned}
$$

The first inequality can be naturally extended to norms in the half-space:

$$
{ }^{\prime}\left\|A(D, \varepsilon) u ; \mathbb{R}_{+}^{n}\right\|_{r-2 m, s-2 \mu} \leq \text { const }^{\prime}\left\|u ; \mathbb{R}_{+}^{n}\right\|_{r, s} .
$$

From the second inequality one can deduce the estimate of the form

$$
{ }^{\prime}\left\|B_{j}(D, \varepsilon) u ; \mathcal{H}^{r-b_{j}-1 / 2, s-\beta_{j}-1 / 2}\left(\mathbb{R}^{n-1}\right)\right\| \leq \mathrm{const}^{\prime}\left\|u ; \mathbb{R}_{+}^{n}\right\|_{r, s} .
$$

Therefore, with the boundary value problems (4.1) we can associate the following continuous operator:

$$
\begin{gathered}
H^{r, s}\left(\mathbb{R}_{+}^{n}\right) \rightarrow H^{r-2 m, s-2 \mu}\left(\mathbb{R}_{+}^{n}\right) \times \prod_{i=1}^{m} H^{r-b_{j}-1 / 2, s-\beta_{j}-1 / 2} \\
\left(u(x) \longrightarrow\left(A(D, \varepsilon) u(x),\left(B_{1}(D, \varepsilon) u\right)\left(x^{\prime}, 0\right), \ldots,\left(B_{m}(D, \varepsilon) u\right)\left(x^{\prime}, 0\right)\right)\right) .
\end{gathered}
$$

Our goal is to find necessary and sufficient conditions for the existence of an estimate for the norm of the operator inverse to (4.5), i.e., an a priori estimate

$$
\begin{aligned}
& \quad\left\|u ; \mathbb{R}_{+}^{n}\right\|_{r, s} \leq \mathrm{const}\left({ }^{\prime}\left\|A(D, \varepsilon) u ; \mathbb{R}_{+}^{n}\right\|_{r-2 m, s-2 \mu}+\left\|u ; \mathbb{R}_{+}^{n}\right\|\right. \\
& \left.+\sum_{j=1}^{m} '\left\|B_{j}(D, \varepsilon) u ; \mathcal{H}^{r-b_{j}-1 / 2, s-\beta_{j}-1 / 2}\left(\mathbb{R}^{n-1}\right)\right\|\right) .
\end{aligned}
$$

Let us recall one more time that the number $s$ in (4.6) is nonnegative, and the necessity of considering the norms with the prime is related to the estimate of the right-hand side of (4.6) for $s<2 \mu$. In this case in the right-hand side it is natural to take the norm of the form (3.20). 
4.2. The statement of the results. The main result of the present paper is the following theorem.

Theorem 4.1. For the problem (4.1) the following conditions are equivalent:

(i) Conditions (A), (B), $\left(\mathrm{C}_{1}\right),\left(\mathrm{C}_{2}\right),\left(\mathrm{C}_{3}\right)$ in Subsection 2.3 hold.

(ii) Conditions (A), (B) in Subsection 2.3, and inequality (2.14) hold.

(iii) Let $r$ and $s$ be natural numbers satisfying (4.2) and the condition

$$
\beta_{\mu}+\frac{1}{2} \leq s<\beta_{\mu+1}+\frac{1}{2}
$$

Then (4.6) holds.

The implication (i) $\rightarrow$ (ii) was established in Theorem 2.6. The main part of this section is devoted to the proof of the estimate (4.6), i.e., the implication (ii) $\rightarrow$ (iii). At the end of the section we will prove the necessity of Conditions $(A),(B),\left(C_{1}\right),\left(C_{2}\right),\left(C_{3}\right)$ for the estimate (4.6) to hold, i.e., the implication (iii) $\rightarrow(\mathrm{i})$.

Remark 4.2. Simultaneously we have established that under the hypotheses of Theorem 2.6. Conditions $\left(\mathrm{C}_{1}\right),\left(\mathrm{C}_{2}\right),\left(\mathrm{C}_{3}\right)$ are equivalent to the estimates (2.14).

4.3. Estimates for the solutions of (4.1) in the case $f(x) \equiv 0$.

Proposition 4.3. Let the problem (4.1) satisfy Conditions (A) and (B) in Subsection 2.3. Let solutions of the problem (2.12) satisfy (2.14). Then for the homogeneous problem

$$
\begin{aligned}
A\left(D^{\prime}, D_{n}, \varepsilon\right) u(x) & =0, \quad x_{n}>0, \\
\left(B_{j}\left(D^{\prime}, D_{n}, \varepsilon\right) u\right)\left(x^{\prime}, 0\right) & =g_{j}\left(x^{\prime}\right), \quad j=1, \ldots, m,
\end{aligned}
$$

we have

$$
\left\|u ; \mathbb{R}_{+}^{n}\right\|_{r, s} \leq \mathrm{const}\left(\left\|u ; \mathbb{R}_{+}^{n}\right\|+\sum_{j=1}^{m} \prime\left\|B_{j}(D, \varepsilon) u ; \mathcal{H}^{r-b_{j}-1 / 2, s-\beta_{j}-1 / 2}\left(\mathbb{R}^{n-1}\right)\right\|\right) .
$$

Proof. The norm in the left-hand side of (4.9) can be replaced by the equivalent norm (3.9), and after that (4.9) is reduced to the following family of inequalities for $\ell=$ $0,1, \ldots, r$ :

$$
\begin{aligned}
& \int_{0}^{\infty}\left\|\Xi_{r-\ell, s-\ell}\left(D^{\prime}, \varepsilon\right) D_{n}^{\ell} u\left(\cdot, x_{n}\right) ; \mathbb{R}^{n-1}\right\|^{2} d x_{n} \\
& \quad \leq \mathrm{const} \sum_{j=1}^{m} \prime\left\|B_{j}(D, \varepsilon) u ; \mathcal{H}^{r-b_{j}-1 / 2, s-\beta_{j}-1 / 2}\left(\mathbb{R}^{n-1}\right)\right\|^{2} .
\end{aligned}
$$

Applying the partial Fourier transform in variables $x^{\prime}$ we reduce the proof of (4.10) to the estimates of the integrands:

$$
\begin{aligned}
\Xi_{r-\ell, s-\ell}^{2}\left(\xi^{\prime}, \varepsilon\right) & \int_{0}^{\infty}\left|D_{n}^{\ell} \hat{u}^{\prime}\left(\xi^{\prime}, x_{n}\right)\right|^{2} d x_{n} \\
& \leq \mathrm{const} \sum_{j=1}^{m} \Xi_{r-b_{j}-1 / 2, s-\beta_{j}-1 / 2}^{2}\left(\xi^{\prime}, \varepsilon\right)\left|g_{j}^{\prime}\left(\xi^{\prime}\right)\right|^{2},
\end{aligned}
$$

where $\hat{u}^{\prime}\left(\xi^{\prime}, x_{n}\right)$ denotes the partial Fourier transform of the function $u(x)$ in the variables $x^{\prime}$.

The functions $\hat{u}^{\prime}\left(\xi^{\prime}, x_{n}\right)$ are solutions of the problem (2.5) and according to Condition (B), they can be represented in the form

$$
\hat{u}^{\prime}\left(\xi^{\prime}, x_{n}\right)=\sum_{j=1}^{m} g_{j}^{\prime}\left(\xi^{\prime}\right) v_{j}\left(x_{n}, \xi^{\prime}, \varepsilon\right)
$$


where $v_{j}\left(x_{n}, \xi^{\prime}, \varepsilon\right)$ is the solution of the problem (2.12). Therefore, the estimate (4.11) is an immediate consequence of the following result.

Lemma 4.4. Let the hypotheses of Proposition 4.3 hold. Then

$$
\left(\int_{0}^{\infty}\left|D_{n}^{\ell} v_{j}\left(x_{n}, \xi^{\prime}, \varepsilon\right)\right|^{2} d x_{n}\right)^{1 / 2} \leq \mathrm{const} \frac{\Xi_{r-b_{j}-1 / 2, s-\beta_{j}-1 / 2}\left(\xi^{\prime}, \varepsilon\right)}{\Xi_{r-\ell, s-\ell}\left(\xi^{\prime}, \varepsilon\right)}, \quad \ell=0,1, \ldots
$$

Proof. Using (3.4) and (4.7) we can rewrite (4.12) as follows:

$$
\begin{aligned}
& \left(\int_{0}^{\infty}\left|D_{n}^{\ell} v_{j}\left(x_{n}, \xi^{\prime}, \varepsilon\right)\right|^{2} d x_{n}\right)^{1 / 2} \\
& \quad \leq \mathrm{const} \begin{cases}\left|\xi^{\prime}\right|{ }^{\ell-\beta_{j}-\frac{1}{2}}\left(1+\varepsilon\left|\xi^{\prime}\right|\right)^{\beta_{j}-b_{j}}, & s-\beta_{j}-\frac{1}{2} \geq 0, \ell \leq s ; \\
\varepsilon^{s-\ell}\left|\xi^{\prime}\right|^{s-\beta_{j}-\frac{1}{2}}\left(1+\varepsilon\left|\xi^{\prime}\right|\right)^{\beta_{j}-b_{j}+\ell-s}, & s-\beta_{j}-\frac{1}{2} \geq 0, \ell>s ; \\
\varepsilon^{\beta_{j}+\frac{1}{2}-s}\left|\xi^{\prime}\right|^{\ell-s}\left(1+\varepsilon\left|\xi^{\prime}\right|\right)^{s-b_{j}-\frac{1}{2}}, & s-\beta_{j}-\frac{1}{2}<0, \ell \leq s ; \\
\varepsilon^{\beta_{j}+\frac{1}{2}-\ell}\left(1+\varepsilon\left|\xi^{\prime}\right|\right)^{\ell-b_{j}-\frac{1}{2}}, & s-\beta_{j}-\frac{1}{2}<0, \ell>s .\end{cases}
\end{aligned}
$$

For the proof, it suffices to verify that the right-hand sides in (2.14) can be estimated by the corresponding right-hand sides in (4.13).

Let $s-\beta_{j}-1 / 2 \geq 0$ and $\ell \leq s$. According to (4.7), this implies $j \leq \mu$ and $\ell \leq \beta_{\mu+1}$. In this case the left-hand side in (4.13) can be estimated by the expression in the top line in (2.14), which is equal to the corresponding line in (4.13).

If $\ell>s$ and $s-\beta_{j}-1 / 2 \geq 0$, then $\ell \geq \beta_{\mu+1}$ and the left-hand side in (4.13) can be estimated by the expression in the second line in (2.14). The ratio of this expression to the expression in the second line in the right-hand side of (4.13) equals

$$
\varepsilon^{\beta_{\mu+1}+\frac{1}{2}-s}\left|\xi^{\prime}\right|^{\beta_{\mu+1}+\frac{1}{2}-s}\left(1+\varepsilon\left|\xi^{\prime}\right|\right)^{s-\beta_{\mu+1}-\frac{1}{2}} \leq 1 .
$$

According to (4.7), the condition $s-\beta_{j}-1 / 2<0$ implies $j>\mu$. Then the condition $\ell \leq s$ implies $\ell \leq \beta_{\mu}$. In this case the left-hand side in (4.13) can be estimated by the expression in the third line in (2.14). The ratio of this expression to the expression in the third line in the right-hand side of (4.13) equals

$$
\varepsilon^{s-\beta_{\mu}-\frac{1}{2}}\left|\xi^{\prime}\right|^{s-\beta_{\mu}-\frac{1}{2}}\left(1+\varepsilon\left|\xi^{\prime}\right|\right)^{\beta_{\mu}+\frac{1}{2}-s} \leq 1 .
$$

Finally, if $s-\beta_{j}-1 / 2<0$ and $\ell>s$, the left-hand side of (4.13) can be estimated by the expression in the fourth line in (2.14), which coincides with the fourth line in the right-hand side of (4.13).

4.4. The proof of (4.6) in the general case. To deduce (4.6) from (4.9) we basically repeat standard arguments used in the theory of elliptic problems. Let us emphasize the role of the norms (3.17) in the case $s<2 \mu$.

Applying the partial Fourier transform, we obtain

$$
\begin{aligned}
A\left(\xi^{\prime}, D_{n}, \varepsilon\right) \hat{u}^{\prime}\left(\xi^{\prime}, x_{n}\right) & =\hat{f}^{\prime}\left(\xi^{\prime}, x_{n}\right), \quad x_{n}>0, \\
B_{j}\left(\xi^{\prime}, D_{n}, \varepsilon\right) \hat{u}^{\prime}\left(\xi^{\prime}, 0\right) & =\hat{g}_{j}^{\prime}\left(\xi^{\prime}\right), \quad j=1, \ldots, m .
\end{aligned}
$$

Let us represent $\hat{u}^{\prime}\left(\xi^{\prime}, x_{n}\right)$ in the form

$$
\hat{u}^{\prime}\left(\xi^{\prime}, x_{n}\right)=v\left(\xi^{\prime}, x_{n}\right)+w\left(\xi^{\prime}, x_{n}\right),
$$

where

$$
A\left(\xi^{\prime}, D_{n}, \varepsilon\right) v\left(\xi^{\prime}, x_{n}\right)=\hat{f}^{\prime}\left(\xi^{\prime}, x_{n}\right), \quad x_{n}>0 .
$$


Then $w\left(\xi^{\prime}, x_{n}\right)$ is the solution of the following problem on the half-line:

$$
\begin{aligned}
& A\left(\xi^{\prime}, D_{n}, \varepsilon\right) w\left(\xi^{\prime}, x_{n}\right)=0, \quad x_{n}>0, \\
& B_{j}\left(\xi^{\prime}, D_{n}, \varepsilon\right) w\left(\xi^{\prime}, 0\right)=\hat{g}_{j}^{\prime}\left(\xi^{\prime}\right)-\chi_{j}\left(\xi^{\prime}\right), \quad j=1, \ldots, m,
\end{aligned}
$$

where

$$
\chi_{j}\left(\xi^{\prime}\right)=B_{j}\left(\xi^{\prime}, D_{n}, \varepsilon\right) v\left(\xi^{\prime}, 0\right) .
$$

We choose a function $v\left(\xi^{\prime}, x_{n}\right)$ satisfying (4.15) and such that

$$
\begin{aligned}
& \Xi_{r-\ell, s-\ell}^{2}\left(\xi^{\prime}, \varepsilon\right) \int_{0}^{\infty}\left|D_{n}^{\ell} v\left(\xi^{\prime}, x_{n}\right)\right|^{2} d x_{n} \\
& \leq \mathrm{const} \int_{0}^{\infty}\left|\left(\varepsilon D_{n}-i \sqrt{1+\varepsilon^{2}\left|\xi^{\prime}\right|^{2}}\right)^{r-2 m-s+2 \mu}\left(D_{n}-i\left|\xi^{\prime}\right|\right)^{s-2 \mu} \hat{f}^{\prime}\left(\xi^{\prime}, x_{n}\right)\right|^{2} d x_{n} ;
\end{aligned}
$$

$$
\begin{aligned}
& \Xi_{r-b_{j}-1 / 2, s-\beta_{j}-1 / 2}^{2}\left(\xi^{\prime}, \varepsilon\right)\left|\chi_{j}^{\prime}\left(\xi^{\prime}\right)\right|^{2} \\
& \leq \mathrm{const} \int_{0}^{\infty}\left|\left(\varepsilon D_{n}-i \sqrt{1+\varepsilon^{2}\left|\xi^{\prime}\right|^{2}}\right)^{r-2 m-s+2 \mu}\left(D_{n}-i\left|\xi^{\prime}\right|\right)^{s-2 \mu} \hat{f}^{\prime}\left(\xi^{\prime}, x_{n}\right)\right|^{2} d x_{n} .
\end{aligned}
$$

Applying (4.10) to $w\left(\xi^{\prime}, x_{n}\right)$ and using (4.17) and (4.16) we arrive at the estimate

$$
\begin{aligned}
& \Xi_{r-\ell, s-\ell}^{2}\left(\xi^{\prime}, \varepsilon\right) \int_{0}^{\infty}\left|D_{n}^{\ell} u\left(\xi^{\prime}, x_{n}\right)\right|^{2} d x_{n} \\
& \leq \operatorname{const} \int_{0}^{\infty}\left|\left(\varepsilon D_{n}-i \sqrt{1+\varepsilon^{2}\left|\xi^{\prime}\right|^{2}}\right)^{r-2 m-s+2 \mu}\left(D_{n}-i\left|\xi^{\prime}\right|\right)^{s-2 \mu} \hat{f}^{\prime}\left(\xi^{\prime}, x_{n}\right)\right|^{2} d x_{n} \\
& \quad+\operatorname{const} \sum_{j=1}^{m} \Xi_{r-b_{j}-1 / 2, s-\beta_{j}-1 / 2}^{2}\left(\xi^{\prime}, \varepsilon\right)\left|g_{j}^{\prime}\left(\xi^{\prime}\right)\right|^{2} .
\end{aligned}
$$

Integrating this inequality with respect to $\xi^{\prime}$ and summing with respect to $\ell$ we arrive at (4.6).

For the function $v\left(\xi^{\prime}, x_{n}\right)$ we take the function

$$
v\left(\xi^{\prime}, x_{n}\right)=R A^{-1}\left(\xi^{\prime}, D_{n}, \varepsilon\right) E \hat{f}^{\prime}\left(\xi^{\prime}, x_{n}\right) .
$$

Here $E$ is the operator of extension from the half-line to the entire line (the Hestenes extension), $R$ the operator of restriction to the half-line, $A^{-1}\left(\xi^{\prime}, D_{n}, \varepsilon\right)$ the pseudodifferential operator with the symbol $A^{-1}\left(\xi^{\prime}, \xi_{n}, \varepsilon\right)$. Equality (4.15) follows from the fact that the differential operator $A\left(\xi^{\prime}, D_{n}, \varepsilon\right)$ commutes with the restriction $R$.

Now we prove the estimates (4.16). Let us denote by $\hat{v}(\xi)$ and $\widehat{E f}(\xi)$ the Fourier transforms with respect to $x_{n}$ of the functions $v\left(\xi^{\prime}, x_{n}\right)$ and $E \hat{f}^{\prime}\left(\xi^{\prime}, x_{n}\right)$. Substituting in the left-hand side of (4.16) the function (4.18), replacing the integral over the half-line with the integral over the entire line, and using the Parceval formula, we estimate the left-hand side of (4.16) by

$$
\int_{-\infty}^{+\infty}\left(1+\varepsilon^{2}\left|\xi^{\prime}\right|^{2}\right)^{r-s}\left|\xi^{\prime}\right|^{2(s-\ell)}\left|A^{-1}(\xi, \varepsilon) \xi_{n}^{\ell}\right|^{2}|\widehat{E f}(\xi)|^{2} d \xi_{n}
$$

for $s \geq \ell$ and by

$$
\int_{-\infty}^{+\infty} \varepsilon^{2(\ell-s)}\left(1+\varepsilon^{2}\left|\xi^{\prime}\right|^{2}\right)^{r-\ell}\left|A^{-1}(\xi, \varepsilon) \xi_{n}^{\ell}\right|^{2}|\widehat{E f}(\xi)|^{2} d \xi_{n}
$$

for $s \leq \ell$. 
In the first case the factor in front of $|\widehat{E f}(\xi)|^{2}$ in the integrand does not exceed

$$
\left[\text { const }\left(1+\varepsilon^{2}\left|\xi^{\prime}\right|^{2}\right)^{r-s}\left(1+\varepsilon^{2}|\xi|^{2}\right)^{-2 m+2 \mu}\right]\left[\left|\xi^{\prime}\right|^{2(s-\ell)}\left|\xi_{n}\right|^{2 \ell}|\xi|^{-4 \mu}\right] \text {. }
$$

Since $r>s$, the first factor does not exceed const $\left(1+\varepsilon^{2}|\xi|^{2}\right)^{r-2 m-s+2 \mu}$. Since $s \geq \ell$, the second factor does not exceed $|\xi|^{2(s-2 \mu)}$. Hence, the factor we are interested in can be estimated by

$$
\text { const }\left|\left(\varepsilon \xi_{n}-i \sqrt{1+\varepsilon^{2}\left|\xi^{\prime}\right|^{2}}\right)^{2(r-2 m-s+2 \mu)}\left(\xi_{n}-i\left|\xi^{\prime}\right|\right)^{2(s-2 \mu)}\right| .
$$

Substituting this estimate in (4.19) and taking into account that

$$
\begin{aligned}
\int_{-\infty}^{+\infty}\left|D_{n}^{\ell} \widehat{\operatorname{Ef}}(\xi)\right|^{2} d \xi_{n} & =\int_{-\infty}^{+\infty}\left|D_{n}^{\ell} E \hat{f}^{\prime}\left(\xi^{\prime}, x_{n}\right)\right|^{2} d x_{n} \\
& \leq \mathrm{const} \int_{0}^{\infty}\left|D_{n}^{\ell} \hat{f}^{\prime}\left(\xi^{\prime}, x_{n}\right)\right|^{2} d x_{n}
\end{aligned}
$$

we prove (4.16) in the case $s \geq \ell$.

In the case $\ell>s$ the factor in front of $|\widehat{E f}(\xi)|^{2}$ in the integrand in (4.19) does not exceed

$$
\text { const }\left[\varepsilon^{2(\ell-s)}\left|\xi_{n}\right|^{2(\ell-s)}\left(1+\varepsilon^{2}\left|\xi^{\prime}\right|^{2}\right)^{r-\ell}\left(1+\varepsilon^{2}|\xi|^{2}\right)^{-2 m+2 \mu}\right]\left[\left|\xi_{n}\right|^{2 s}|\xi|^{-4 \mu}\right] \text {. }
$$

The first factor in this formula can be estimated by const $\left(1+\varepsilon^{2}|\xi|^{2}\right)^{r-2 m-s+2 \mu}$ and the second by $|\xi|^{2(s-2 \mu)}$; hence, we again arrive at (4.16).

We now pass to the verification of (4.17). Let us note that

$$
\chi_{j}\left(\xi^{\prime}\right)=\int_{-\infty}^{+\infty} \frac{B_{j}(\xi, \varepsilon)}{A(\xi, \varepsilon)} \widehat{E f}(\xi) d \xi_{n}:=\int_{-\infty}^{+\infty} H_{j}(\xi, \varepsilon) F(\xi, \varepsilon) d \xi_{n},
$$

where we denoted

$$
\begin{aligned}
F(\xi, \varepsilon) & =\left(\varepsilon \xi_{n}-i \sqrt{1+\varepsilon^{2}\left|\xi^{\prime}\right|^{2}}\right)^{r-2 m-s+2 \mu}\left(\xi_{n}-i\left|\xi^{\prime}\right|\right)^{s-2 \mu} \widehat{E} f(\xi), \\
H_{j}(\xi, \varepsilon) & =B_{j}(\xi, \varepsilon) A^{-1}(\xi, \varepsilon)\left(\varepsilon \xi_{n}-i \sqrt{1+\varepsilon^{2}\left|\xi^{\prime}\right|^{2}}\right)^{-r+2 m+s-2 \mu}\left(\xi_{n}-i\left|\xi^{\prime}\right|\right)^{-s+2 \mu} .
\end{aligned}
$$

By the Schwarz inequality,

$$
\left|\chi_{j}\left(\xi^{\prime}\right)\right|^{2} \leq \int_{-\infty}^{+\infty}\left|H_{j}(\xi, \varepsilon)\right|^{2} d \xi_{n} \int_{-\infty}^{+\infty}|F(\xi, \varepsilon)|^{2} d \xi_{n}
$$

and the proof of (4.17) is reduced to the verification of the inequality

$$
\Xi_{r-b_{j}-1 / 2, s-\beta_{j}-1 / 2}^{2}\left(\xi^{\prime}, \varepsilon\right) \int_{-\infty}^{+\infty}\left|H_{j}(\xi, \varepsilon)\right|^{2} d \xi_{n} \leq \mathrm{const},
$$

where the right-hand side is independent of $\xi^{\prime}$ and $\varepsilon$.

The function $\left|H_{j}(\xi, \varepsilon)\right|^{2}$ can be estimated as follows:

$$
\left|H_{j}(\xi, \varepsilon)\right|^{2} \leq \text { const }\left(1+\varepsilon^{2}|\xi|^{2}\right)^{-r+s-b_{j}+\beta_{j}}|\xi|^{-2 s+2 \beta_{j}} .
$$

Therefore, we need to verify that

$$
\Xi_{r-b_{j}-1 / 2, s-\beta_{j}-1 / 2}^{2}\left(\xi^{\prime}, \varepsilon\right) \int_{-\infty}^{+\infty}\left(1+\varepsilon^{2}|\xi|^{2}\right)^{-r+s-b_{j}+\beta_{j}}|\xi|^{-2 s+2 \beta_{j}} d \xi_{n} \leq \mathrm{const} .
$$

First, let us consider the case $s-\beta_{j}-1 / 2>0$. In this case the left-hand side of (4.21) becomes

$$
\left(1+\varepsilon^{2}\left|\xi^{\prime}\right|^{2}\right)^{r-s-b_{j}+\beta_{j}}\left|\xi^{\prime}\right|^{2 s-2 \beta_{j}-1} \int_{-\infty}^{+\infty}\left(1+\varepsilon^{2}|\xi|^{2}\right)^{-r+s-b_{j}+\beta_{j}}|\xi|^{-2 s+2 \beta_{j}} d \xi_{n} .
$$


Since $r-s-b_{j}+\beta_{j} \geq 0$ by (4.2), our expression can be estimated by

$$
\left|\xi^{\prime}\right|^{2 s-2 \beta_{j}-1} \int_{-\infty}^{+\infty}\left(\left|\xi^{\prime}\right|^{2}+\xi_{n}^{2}\right)^{-s+\beta_{j}} d \xi_{n}=\text { const . }
$$

(We used the substitution $\xi_{n}=\left|\xi^{\prime}\right| t$.)

In the case $s-\beta_{j}-1 / 2 \leq 0$ the left-hand side of (4.21) takes the form

$$
\varepsilon^{2 \beta_{j}-2 s+1}\left(1+\varepsilon^{2}\left|\xi^{\prime}\right|^{2}\right)^{r-b_{j}-1 / 2} \int_{-\infty}^{+\infty}\left(1+\varepsilon^{2}|\xi|^{2}\right)^{-r+s-b_{j}+\beta_{j}}|\xi|^{-2 s+2 \beta_{j}} d \xi_{n} .
$$

Similarly to what was done in analyzing (3.15), we make the substitution $\xi_{n}=\varepsilon^{-1}(1+$ $\left.\varepsilon^{2}\left|\xi^{\prime}\right|^{2}\right)^{1 / 2} t$ and after some elementary calculations obtain

$$
\int_{-\infty}^{\infty}\left(\frac{\varepsilon^{2}+\varepsilon^{2}\left|\xi^{\prime}\right|^{2}}{1+\varepsilon^{2}\left|\xi^{\prime}\right|^{2}}+t^{2}\right)^{\beta_{j}-s}\left(1+t^{2}\right)^{-r+s-\beta_{j}+b_{j}} d t
$$

Since $s$ and $\beta_{j}$ are integers, $s-\beta_{j} \geq-1 / 2$ implies $s-\beta_{j} \geq 0$, so that the latter integral does not exceed

$$
\int_{-\infty}^{\infty}\left(1+t^{2}\right)^{-r+b_{j}} d t=\text { const }
$$

(We used the last inequality in (4.2).)

4.5. The necessity of Conditions (A), (B), $\left(\mathrm{C}_{1}\right),\left(\mathrm{C}_{2}\right),\left(\mathrm{C}_{3}\right)$. The proof of necessity in a case similar to the one considered here was given in 14. Therefore, we will briefly describe only the main steps of the proof.

The necessity of Condition (A). Substituting in (4.6) a smooth function $u$ supported in $\mathbb{R}_{+}^{n}$ we obtain the inequality

$$
\int\left[|\xi|^{2 s}\left(1+\varepsilon^{2}|\xi|^{2}\right)^{r-s}-C^{2}|\xi|^{2 s-2 \mu}\left(1+\varepsilon^{2}|\xi|^{2}\right)^{r-s-2 m+2 \mu}|A(\xi, \varepsilon)|^{2}\right]|\hat{u}(\xi)|^{2} d \xi \leq 0 .
$$

Since $u$ is arbitrary, we arrive at (1.2).

The necessity of other conditions is based on the following inequality on the half-line:

$$
\begin{aligned}
\text { const } & \sum_{\ell=0}^{r} \Xi_{r-\ell, s-\ell}^{2}\left(\xi^{\prime}, \varepsilon\right) \int_{0}^{\infty}\left|D_{n}^{\ell} V\left(x_{n}\right)\right|^{2} d x_{n} \\
\leq & \int_{0}^{\infty}\left|R\left(D_{n}-i\left|\xi^{\prime}\right|\right)^{s-2 \mu}\left(\varepsilon D_{n}-i \sqrt{1+\varepsilon^{2}\left|\xi^{\prime}\right|^{2}}\right)^{r-s-2 m+2 \mu} A\left(\xi^{\prime}, D_{n}, \varepsilon\right) L V\right|^{2} d x_{n} \\
& +\sum_{j=1}^{m} \Xi_{r-b_{j}-1 / 2, s-\beta_{j}-1 / 2}^{2}\left(\xi^{\prime}, \varepsilon\right)\left|B_{j}\left(\xi^{\prime}, D_{n}, \varepsilon\right) V(0)\right|^{2} .
\end{aligned}
$$

This inequality is deduced from (4.6), where we set $u(x)=\phi\left(x^{\prime}\right) V\left(x_{n}\right)$. For details, see [16. Chapter 3, Proposition 2 in 2.3].

The necessity of Condition (B). Let us assume that $V\left(x_{n}\right) \in L_{2}\left(\mathbb{R}_{+}\right)$is a solution of the homogeneous equation

$$
A\left(\xi^{\prime}, D_{n}, \varepsilon\right) V\left(x_{n}\right)=0, \quad x_{n}>0 .
$$

Then $V$ also satisfies the equation

$$
A^{+}\left(\xi^{\prime}, D_{n}, \varepsilon\right) V\left(x_{n}\right)=0, \quad x_{n}>0,
$$

and the estimate (4.22) yields the inequality

$$
c\left(\xi^{\prime}, \varepsilon\right) \sum_{l=0}^{r}\left\|D_{n}^{l} V\right\|_{L_{2}\left(\mathbb{R}_{+}\right)} \leq \sum_{j=1}^{m}\left|\bar{B}_{j}\left(\xi^{\prime}, \varepsilon, D_{n}\right) V(0)\right|,
$$


where $\bar{B}_{j}$ is the remainder in the division of $B_{j}$ by $A^{+}$and $c\left(\xi^{\prime}, \varepsilon\right)>0$ for $\xi^{\prime} \neq 0$ and $\varepsilon \geq 0$.

A standard estimate for the trace of a function on $\mathbb{R}_{+}$implies that

$$
\sum_{j=1}^{r}\left|D_{n}^{j-1} V(0)\right| \leq C \sum_{j=1}^{r+1}\left\|D_{n}^{j-1} V\right\|_{L_{2}\left(\mathbb{R}_{+}\right)} .
$$

Since $r \geq m$, we get

$$
\tilde{c}\left(\xi^{\prime}, \varepsilon\right) \sum_{j=1}^{m}\left|D_{n}^{j-1} V(0)\right| \leq \sum_{j=1}^{m}\left|\sum_{k=1}^{m} \bar{b}_{j k}\left(\xi^{\prime}, \varepsilon\right) D_{n}^{k-1} V(0)\right|,
$$

where (as in Section 2)

$$
\bar{B}_{j}\left(\xi^{\prime}, \varepsilon, z\right)=\sum_{k=1}^{m} \bar{b}_{j k}\left(\xi^{\prime}, \varepsilon\right) z^{k-1} .
$$

In the latter inequality, the constant $\tilde{c}\left(\xi^{\prime}, \varepsilon\right)$ is positive for $\xi^{\prime} \neq 0$.

Let us note that the Cauchy problem

$$
D_{n}^{k-1} V(0)=\zeta_{k}, \quad k=1, \ldots, m,
$$

for the ordinary differential equation (4.23) has a unique solution for each vector $\zeta=$ $\left(\zeta_{1}, \ldots, \zeta_{m}\right) \in \mathbb{C}^{m}$. We have the inequality

$$
\tilde{c}\left(\xi^{\prime}, \varepsilon\right)|\zeta| \leq\left|\mathcal{B}\left(\xi^{\prime}, \varepsilon\right) \zeta\right|, \quad \mathcal{B}\left(\xi^{\prime}, \varepsilon\right):=\left(\bar{b}_{j k}\left(\xi^{\prime}, \varepsilon\right)\right)_{j, k=1, \ldots, m},
$$

which means that the matrix $\mathcal{B}\left(\xi^{\prime}, \varepsilon\right)$ is nonsingular for $\left|\xi^{\prime}\right| \neq 0, \varepsilon \geq 0$; i.e., the necessity of Condition (B) is proved.

The necessity of Condition $\left(\mathrm{C}_{2}\right)$. In (4.22) take $\varepsilon=0$. Taking into account (3.4) and (4.7) we obtain

$$
\Xi_{r-b_{j}-1 / 2, s-\beta_{j}-1 / 2}\left(\xi^{\prime}, 0\right)= \begin{cases}\left|\xi^{\prime}\right|^{s-\beta_{j}-1 / 2}, & j \leq \mu, \\ 0, & j>\mu .\end{cases}
$$

Hence (4.22) takes the form

$$
\begin{aligned}
\text { const } & \sum_{\ell=0}^{r}\left|\xi^{\prime}\right|^{2(s-\ell)} \int_{0}^{\infty}\left|D_{n}^{\ell} V\left(x_{n}\right)\right|^{2} d x_{n} \\
\leq & \int_{0}^{\infty}\left|R\left(D_{n}-i\left|\xi^{\prime}\right|\right)^{s-2 \mu} A_{2 \mu}\left(\xi^{\prime}, D_{n}\right) L V\right|^{2} d x_{n} \\
& +\sum_{j=1}^{\mu}\left|\xi^{\prime}\right|^{2\left(s-\beta_{j}-1 / 2\right)}\left|B_{\beta_{j}}\left(\xi^{\prime}, D_{n}\right) V(0)\right|^{2} .
\end{aligned}
$$

Using the arguments similar to those applied previously, we can prove that the system of operators $\left\{A_{2 \mu}, B_{\beta_{1}}, \ldots, B_{\beta_{\mu}}\right\}$ satisfies the Shapiro-Lopatinskii conditions.

The necessity of Condition $\left(\mathrm{C}_{1}\right)$. To verify this condition, let us multiply both sides of (4.22) by $\varepsilon^{s-r}$ and pass to the limit as $\varepsilon \rightarrow \infty$. We obtain

$$
\begin{aligned}
\text { const } & \sum_{\ell=0}^{r}\left|\xi^{\prime}\right|^{2(r-\ell)} \int_{0}^{\infty}\left|D_{n}^{\ell} V\left(x_{n}\right)\right|^{2} d x_{n} \\
\leq & \int_{0}^{\infty}\left|\left(D_{n}-i\left|\xi^{\prime}\right|\right)^{r-2 m} A_{2 m}\left(\xi^{\prime}, D_{n}\right) V\left(x_{n}\right)\right|^{2} d x_{n} \\
& +\sum_{j=1}^{m}\left|\xi^{\prime}\right|^{2\left(r-b_{j}-1 / 2\right)}\left|B_{j, b_{j}}\left(\xi^{\prime}, D_{n}\right) V(0)\right|^{2} .
\end{aligned}
$$

This implies the necessity of $\left(\mathrm{C}_{1}\right)$. 
The necessity of Condition $\left(\mathrm{C}_{3}\right)$ can be deduced from the inequality

$$
\begin{aligned}
\text { const } & \sum_{l=s}^{r} \int_{0}^{\infty}\left|D_{n}^{\ell} V\left(x_{n}\right)\right|^{2} d x_{n} \\
\leq & \int_{0}^{\infty}\left|\left(D_{n}-i\right)^{r-s-2 m+2 \mu} D_{n}^{s} Q\left(D_{n}\right) V\right|^{2} d x_{n}+\sum_{j=\mu+1}^{m}\left|B_{j}\left(0, D_{n}, 1\right) V(0)\right|^{2} .
\end{aligned}
$$

To prove this inequality we replace in (4.22) $\varepsilon$ with $1 / \rho, \xi^{\prime}$ with $\rho^{\gamma} \xi^{\prime}$, where $0<\gamma<1$, and $V\left(x_{n}\right)$ with $\rho^{-s+1 / 2} V\left(\rho x_{n}\right)$. Then the expression

$$
\sum_{\ell=0}^{s-1}\left|\xi^{\prime}\right|^{2 s-2 \ell}\left(1+\varepsilon^{2}\left|\xi^{\prime}\right|^{2}\right)^{r-s} \int_{0}^{\infty}\left|D_{n}^{\ell} V\left(x_{n}\right)\right|^{2} d x_{n}
$$

becomes $O\left(\rho^{-2(1-\gamma)(s-\ell)}\right) \leq O\left(\rho^{-2(1-\gamma)}\right)$, and

$$
\sum_{\ell=s}^{r}|\varepsilon|^{2 \ell-2 s}\left(1+\varepsilon^{2}\left|\xi^{\prime}\right|^{2}\right)^{r-\ell} \int_{0}^{\infty}\left|D_{n}^{\ell} V\left(x_{n}\right)\right|^{2} d x_{n}
$$

becomes

$$
\sum_{l=s}^{r} \int_{0}^{\infty}\left|D_{n}^{\ell} V\left(x_{n}\right)\right|^{2} d x_{n}+O\left(\rho^{-2(1-\gamma)}\right) .
$$

The first term in the right-hand side of (4.22) becomes

$$
\begin{aligned}
\int_{0}^{\infty} \mid R\left(D_{n}-i \rho^{-1+\gamma}\left|\xi^{\prime}\right|\right)^{s-2 \mu}\left(D_{n}-i \rho^{-1} \sqrt{1+\rho^{-2+2 \gamma}\left|\xi^{\prime}\right|^{2}}\right)^{r-s-2 m+2 \mu} & \\
& \times\left. A\left(\rho^{-1+\gamma} \xi^{\prime}, D_{n}, 1\right) L V\left(x_{n}\right)\right|^{2} d x_{n} .
\end{aligned}
$$

Noting that

$$
A\left(0, D_{n}, 1\right)=A_{2 \mu}(0,1) D_{n}^{2 \mu} Q\left(D_{n}\right),
$$

we rewrite the latter expression as

$$
\left|A_{2 \mu}(0,1)\right| \int_{0}^{\infty}\left|\left(D_{n}-i\right)^{r-s-2 m+2 \mu} D_{n}^{s} Q\left(D_{n}\right) V\right|^{2} d x_{n}+O\left(\rho^{-2(1-\gamma)}\right) .
$$

Now consider the last term in the right-hand side of (4.22). The sum

$$
\sum_{j=1}^{\mu}\left|\xi^{\prime}\right|^{s-\beta_{j}-1 / 2}\left(1+\varepsilon^{2}\left|\xi^{\prime}\right|^{2}\right)^{r-b_{j}-1 / 2}\left|B_{j}\left(\xi^{\prime}, D_{n}, \varepsilon\right) V(0)\right|^{2}
$$

becomes

$$
\sum_{j=1}^{\mu} O\left(\rho^{-(1-\gamma)\left(s-\beta_{j}-1 / 2\right)}\right) \leq O\left(\rho^{-(1-\gamma) / 2}\right)
$$

(since $s$ is an integer and from the left inequality (4.7), we see that in fact $s-\beta_{j}-1 / 2 \leq$ $1 / 2$ for $j \leq \mu)$. Next,

$$
\sum_{j=\mu+1}^{m} \varepsilon^{\beta_{j}+1 / 2-s}\left(1+\varepsilon^{2}\left|\xi^{\prime}\right|^{2}\right)^{r-b_{j}-1 / 2}\left|B_{j}\left(\xi^{\prime}, D_{n}, \varepsilon\right) V(0)\right|^{2}
$$

becomes

$$
\sum_{j=\mu+1}^{m}\left(1+\rho^{-2+2 \gamma}\left|\xi^{\prime}\right|^{2}\right)^{r-b_{j}-1 / 2}\left|B_{j}\left(\rho^{-2+2 \gamma} \xi^{\prime}, D_{n}, 1\right) V(0)\right|^{2} .
$$

Taking the limit as $\rho \rightarrow 0$, we obtain inequality (4.26). Condition $\left(\mathrm{C}_{3}\right)$ is an easy consequence of this inequality. For details, see [14]. 


\section{Appendix. Formal asymptotic solution of the problem $(0.1),(0.2)$}

In this appendix we show how to use the the Vishik-Lyusternik method for the construction of a formal asymptotic solution of the problem (0.1), (0.2). This construction illustrates once again the definition of small parameter ellipticity of a boundary problem.

5.1. General comments. We start with a general definition of a formal asymptotic solution (FAS); a detailed discussion of this notion can be found in [19.

Suppose we have an operator equation with the operator depending on a small parameter:

$$
\mathfrak{A}(\varepsilon) u=f .
$$

A formal series

$$
U(\varepsilon)=\sum_{k=k_{0}}^{\infty} \varepsilon^{k} u_{k}(\varepsilon)
$$

is called a formal asymptotic solution of the equation (5.1) if for each $\nu>0$ there exists $N=N(\nu)$ such that the finite sum of the series (5.2),

$$
U_{N}(\varepsilon)=\sum_{k=k_{0}}^{N} \varepsilon^{k} u_{k}(\varepsilon),
$$

is a solution of (5.1) up to $O\left(\varepsilon^{\nu}\right)$, i.e.,

$$
\left[\mathfrak{A}(\varepsilon) U_{N}(\varepsilon)-f(x)\right] \leq \text { const } \varepsilon^{\nu},
$$

where [.] is a norm in the space of solutions of (5.1).

The locality principle that lies in the foundation of the elliptic theory allows us to "glue together" FAS's of the problem (0.1), (0.2) from "local" FAS's defined in neighborhoods of some inner points of the manifold $M$ and some points of the boundary $\partial M$. In other words, we need to discuss the construction of FAS's for the equation (0.1) in $\mathbb{R}^{n}$ and for the problem (0.1), (0.2) in $\mathbb{R}_{+}^{n}$.

The construction of the formal asymptotic solution in $\mathbb{R}^{n}$ is absolutely standard. We are looking for an FAS in the form

$$
U(x, \varepsilon)=\sum_{k=0}^{N} \varepsilon^{k} u_{k}(x) .
$$

Substituting this series in (0.1) and comparing the terms with equal powers of $\varepsilon$ we obtain the recurrence equations

$$
A_{2 \mu} u_{0}=f, \quad A_{2 \mu} u_{1}=-A_{2 \mu+1} u_{0},
$$

and for an arbitrary $k>1$ we obtain

$$
A_{2 \mu} u_{k}=-A_{2 \mu+1} u_{k-1}-\cdots-A_{2 m} u_{k-2 m+2 \mu},
$$

where we formally set $u_{k-l}=0$ for $l>k$.

Equations (5.4), (5.5) show that our recurrence system is solvable provided, e.g., the operator

$$
A_{2 \mu}(x, D): H^{r}(M) \rightarrow H^{r-2 \mu}(M)
$$

has a bounded inverse for some $r$. A necessary condition for such invertibility is the ellipticity of the operator $A_{2 \mu}(x, D)$. In general, this condition is not sufficient and we need additional conditions imposed on the lower terms. To justify the constructed FAS we need an estimate from below for the operator $A(x, D, \varepsilon)$ that would ensure the uniqueness in $\mathbb{R}^{n}$. 
5.2. Formal asymptotic solution in the half-space. The boundary layer method. We will consider the problem (0.1), (0.2) in the half-space

$$
\mathbb{R}_{+}^{n}:=\left\{x=\left(x^{\prime}, x_{n}\right), x^{\prime} \in \mathbb{R}^{n-1}, x_{n} \geq 0\right\} .
$$

We look for a formal solution of this problem in the form

$$
U(x, \varepsilon)+V\left(x^{\prime}, \frac{x_{n}}{\varepsilon}, \varepsilon\right) .
$$

The first term is the so-called exterior expansion of the form (5.3). Elements of this sum are related by the formulas (5.4), (5.5). The second term is the so-called inner expansion, or the boundary layer in the Vishik-Lyusternik form,

$$
V\left(x^{\prime}, \frac{x_{n}}{\varepsilon}, \varepsilon\right)=\sum_{l=0}^{\infty} \varepsilon^{l_{0}+l} v_{l}\left(x^{\prime}, \frac{x_{n}}{\varepsilon}\right) .
$$

The integer $l_{0}$ will be determined later.

We look for the inner expansion (5.7) as a solution of the homogeneous equation

$$
A(x, D, \varepsilon) V\left(x^{\prime}, \frac{x_{n}}{\varepsilon}, \varepsilon\right)=0 .
$$

Substituting $x_{n}$ with $t=x_{n} / \varepsilon$ we rewrite this equation as

$$
\sum_{l=0}^{\infty} \varepsilon^{l_{0}+l-2 \mu}\left(A\left(\varepsilon t, x^{\prime}, \varepsilon D^{\prime}, D_{t}\right) v_{l}\right)\left(x^{\prime}, t\right)=0 .
$$

Expand $A\left(\varepsilon t, x^{\prime}, \varepsilon D^{\prime}, D_{t}\right)$ in a series (in general, formal) in powers of $\varepsilon$,

$$
\begin{aligned}
A\left(\varepsilon t, x^{\prime}, \varepsilon D^{\prime}, D_{n}\right) & =A\left(0, x^{\prime}, 0, D_{t}\right)+\sum_{s=1} \varepsilon^{s} \sum_{p+|\alpha|=s} \frac{t^{p}}{p ! \alpha !}\left(\partial_{t}^{p} \partial_{\xi}^{\alpha} A\right)\left(0, x^{\prime}, 0, D_{t}\right) D^{\prime \alpha} \\
& :=A\left(0, x^{\prime}, 0, D_{t}\right)+\sum_{s=1} \varepsilon^{s} A_{s}\left(t, x^{\prime}, D^{\prime}, D_{t}\right),
\end{aligned}
$$

substitute in (5.9), and compare the terms with equal powers of $\varepsilon$. We obtain a recurrent system of ordinary differential equations in $t$ (parameterized by points of $x^{\prime} \in \mathbb{R}^{n-1}$ ):

$$
A\left(0, x^{\prime}, 0, D_{t}, 1\right) v_{l}\left(x^{\prime}, t\right)=-\sum_{s=1}^{l} A_{s}\left(t, x^{\prime}, D^{\prime}, D_{t}\right) v_{l-s}\left(x^{\prime}, t\right) .
$$

Replacing the variable $t$ with $x_{n}$ we obtain the relations

$$
\begin{aligned}
A_{2 \mu}(x, D) u_{k}(x) & =F_{k}\left(x, u_{0}, \ldots, u_{k-1}\right), \\
A\left(0, x^{\prime}, 0, D_{n}, 1\right) v_{l}\left(x^{\prime}, t\right) & =F_{l}^{\prime}\left(x^{\prime}, v_{0}, \ldots, v_{l-1}\right),
\end{aligned}
$$

which ensure that the function (5.7) is a formal solution of the equation (0.1). Let us note that in the right-hand side of (5.10) and (5.11) we have functions that become known once we determine $u_{0}, \ldots, u_{k-1}$ (respectively, $v_{0}, \ldots, v_{l-1}$ ).

Now we should choose the boundary conditions for the functions $u_{k}, v_{l}$ in such a way that the functions (5.6) formally satisfy the boundary condition (0.2),

$$
\begin{gathered}
\left.B_{j}\left(x^{\prime}, D, \varepsilon\right) U(x, \varepsilon)\right|_{x_{n}=0}+\left.B_{j}\left(x^{\prime}, D, \varepsilon\right) V\left(x^{\prime}, x_{n} / \varepsilon, \varepsilon\right)\right|_{x_{n}=0}=g_{j}, \\
j=1, \ldots, m .
\end{gathered}
$$

An important role is played by the choice of the number $l_{0}$ :

$$
l_{0}=\beta_{\mu+1} \geq \beta_{\mu}+1 .
$$


5.3. The choice of functions $u_{0}$ and $v_{0}$. Applying the boundary operator to the exterior expansion we obtain

$$
\begin{aligned}
& \left.B_{j}\left(x^{\prime}, D, \varepsilon\right) U(x, \varepsilon)\right|_{x_{n}=0} \\
& \quad=\sum_{l=0}^{\infty} \varepsilon^{l}\left[\left(B_{\beta_{j}}\left(x^{\prime}, D\right) u_{l}\right)\left(x^{\prime}, 0\right)+\sum_{s \geq 1} \varepsilon^{s}\left(B_{j s}\left(x^{\prime}, D\right) u_{l-s}\right)\left(x^{\prime}, 0\right)\right] \\
& =B_{\beta_{j}}\left(x^{\prime}, D\right) u_{0}\left(x^{\prime}, 0\right)+O(\varepsilon) .
\end{aligned}
$$

Next, applying the boundary operator to the internal expansion and using (5.13) we obtain

$$
\begin{aligned}
\left.B_{j}\left(x^{\prime}, D, \varepsilon\right) V\left(x^{\prime}, \frac{x_{n}}{\varepsilon}, \varepsilon\right)\right|_{x_{n}=0}=\varepsilon^{-\beta_{j}}\left(B_{j}\left(x^{\prime}, \varepsilon D^{\prime}, D_{n}, 1\right) V\right)\left(x^{\prime}, 0, \varepsilon\right) \\
=\sum_{l=0}^{\infty} \varepsilon^{l+\beta_{\mu+1}-\beta_{j}}\left[\left(B_{j}\left(x^{\prime}, 0, D_{n}, 1\right) v_{l}\left(x^{\prime}, 0\right)\right)+\sum_{s \geq 1} \varepsilon^{s}\left(B_{j s}\left(x^{\prime}, D\right) v_{l-s}\right)\left(x^{\prime}, 0\right)\right] \\
=\varepsilon^{\beta_{\mu+1}-\beta_{j}} B_{j}\left(x^{\prime}, 0, D_{n}, 1\right) v_{0}\left(x^{\prime}, 0\right)+O\left(\varepsilon^{\beta_{\mu+1}-\beta_{j}+1}\right) .
\end{aligned}
$$

For $j \leq \mu$ the right-hand side of (5.15) is of order $O(\varepsilon)$ and does not contribute to the boundary terms of the zeroth (with respect to $\varepsilon$ ) order.

Now we define $u_{0}(x)$ as the solution of the boundary value problem

$$
A_{2 \mu}(x, D) u_{0}(x)=f(x), \quad\left(B_{\beta_{j}}\left(x^{\prime}, D\right) u_{0}\right)\left(x^{\prime}\right)=g_{j}\left(x^{\prime}\right), \quad j=1, \ldots, \mu .
$$

To determine $u_{0}(x)$, the operator

$$
\left\{A_{2 \mu}(x, D), B_{\beta_{1}}\left(x^{\prime}, D\right), \ldots, B_{\beta_{\mu}}\left(x^{\prime}, D\right)\right\}: H^{r}\left(\mathbb{R}_{+}^{n}\right) \rightarrow H^{r-2 \mu}\left(\mathbb{R}_{+}^{n}\right) \times \prod_{j=1}^{\mu} H^{r-\beta_{j}-\frac{1}{2}}\left(\mathbb{R}^{n_{-} 1}\right)
$$

should have a boundary inverse for some $r$. In the case of constant coefficients, we come to Condition $\left(\mathrm{S}_{2}\right)$. In the case of variable coefficients we need additional conditions on the lower terms.

Now we determine the boundary conditions for $v_{0}$. Let us assume that

$$
\beta_{\mu+1}=\cdots=\beta_{\nu}<\beta_{\nu+1}
$$

Then we naturally obtain the equalities

$$
B_{j}\left(x^{\prime}, 0, D_{n}, 1\right) v_{0}\left(x^{\prime}, 0\right)=g_{j}-B_{\beta_{j}}\left(x^{\prime}, D\right) u_{0}, \quad j=\mu+1, \ldots, \nu .
$$

For $j \geq \nu+1$ the leading terms of the expansions (5.15) contain negative powers of $\varepsilon$. It is natural to make the coefficients at these powers equal to zero. Hence, we obtain the additional boundary conditions

$$
B_{j}\left(x^{\prime}, 0, D_{n}, 1\right) v_{0}\left(x^{\prime}, 0\right)=0, \quad j=\nu+1, \ldots, m .
$$

As a result, for $v_{0}$ we obtain the ordinary equation

$$
A\left(0, x^{\prime}, 0, D_{n}, 1\right) v_{0}(x)=0
$$

with the boundary conditions (5.17), (5.18). The solvability of this problem is guaranteed by Condition $\left(\mathrm{S}_{3}\right)$. 
5.4. The choice of further terms of the expansion. Since we already have recurrent equations (5.10), (5.11) for $u_{\ell}, v_{\ell}, \ell \geq 1$, it remains to present recurrent formulas for the boundary conditions. For $j=1, \ldots, \mu$ we have

$$
\left.B_{j} U\right|_{x_{n}=0}-g_{j}=\varepsilon\left(B_{\beta_{j}} u_{1}+B_{j 1} u_{0}\right)+O\left(\varepsilon^{2}\right) .
$$

If $\beta_{\mu+1}-\beta_{j} \geq 2$ for $j \leq \mu$, then we set

$$
B_{\beta_{j}} u_{1}=-B_{j 1} u_{0} .
$$

If $\beta_{\mu+1}-\beta_{j}=1$ for some $j \leq \mu$, we set

$$
B_{\beta_{j}} u_{1}=-B_{j 1} u_{0}-B_{j}\left(x^{\prime}, 0, D_{n}, 1\right) v_{0} .
$$

Let us pass to the boundary conditions for $v_{1}$. For $j=\mu+1, \ldots, \nu$ we have (taking into account the already determined $u_{0}, v_{0}$ )

$$
\left.B_{j} V\right|_{x_{n}=0}+\left.B_{j} U\right|_{x_{n}=0}-g_{j}=\varepsilon\left(B_{j}\left(x^{\prime}, 0, D_{n}, 1\right) v_{1}+B_{j 1} v_{0}+B_{\beta_{j}} u_{1}\right)+O\left(\varepsilon^{2}\right) .
$$

Therefore, it is natural to set (see (5.17))

$$
B_{j}\left(x^{\prime}, 0, D_{n}, 1\right) v_{1}\left(x^{\prime}, 0\right)=-B_{j 1} v_{0}-B_{\beta_{j}} u_{1}, \quad j=\mu+1, \ldots, \nu .
$$

Analyzing the expressions $\left.B_{j} V\right|_{x_{n}=0}+\left.B_{j} U\right|_{x_{n}=0}-g_{j}$ for $j>\nu$ we assume that there exists $\nu^{\prime}$ such that

$$
\beta_{\nu+1}=\cdots=\beta_{\nu^{\prime}}=\beta_{\mu+1}+1 .
$$

Then we set

$$
B_{j}\left(x^{\prime}, 0, D_{n}, 1\right) v_{1}\left(x^{\prime}, 0\right)=g_{j}-B_{\beta_{j}} u_{1}-B_{j 1} v_{0}, \quad j=\nu+1, \ldots, \nu^{\prime} .
$$

Taking into account (5.17) we see that for $j \geq \nu^{\prime}+1$ the leading terms of (5.15) contain negative powers of $\varepsilon$. Hence we set

$$
B_{j}\left(x^{\prime}, 0, D_{n}, 1\right) v_{0}\left(x^{\prime}, 1\right)=0, \quad j=\nu^{\prime}, \ldots, m .
$$

Now we can determine the function $u_{1}$.

Continuing this process, we construct the entire asymptotic series.

Remark 5.1. The above construction shows that even at the first step we satisfy equation (0.1) up to $\varepsilon$. However, to satisfy the boundary conditions (0.2) up to $\varepsilon$ we need sufficiently many terms of the expansions (5.3) and (5.7).

\section{REFERENCES}

[1] M. I. Vishik and L. A. Lyusternik, Regular degeneration and boundary layer for linear differential equations with small parameter. Uspekhi Mat. Nauk 12 (1957), no. 5, 3-122; English transl., Amer. Math. Soc. Transl. (2), vol. 20, Amer. Math. Soc., New York, 1962, pp. 239-364. MR0136861 $(25: 322)$

[2] L. S. Frank, Coercive singular perturbations. I. A priori estimates. Annali Mat. Pura Appl. 119 (1979), 41-113. MR0551218 (81b:35025)

[3] Singular perturbations. I. Spacers and singular perturbations on manifolds without boundary. North Holland, Amsterdam, 1990. MR1071175 (91i:58144)

[4] _ Singular perturbations in elasticity theory. IOS Press, Berlin, 1997. MR1637636 (99h:35010)

[5] S. A. Nazarov, The Vishik-Lyusternik method for elliptic boundary value problems in regions with conic points. I. Problem in a cone. Sibirsk. Mat. Zh. 22 (1981), no. 4, 142-163; English transl., Siberian Math. J. 22 (1981), no. 4, 594-611. MR0624412 (83m:35046a)

[6] _ . The Vishik-Lyusternik method for elliptic boundary value problems in regions with conic points. II. Problem in a bounded domain. Sibirsk. Mat. Zh. 22 (1981), no. 5, 132-152; English transl., Siberian Math. J. 22 (1981), no. 5, 753-769. MR0632823 (83m:35046b)

[7] Justification of asymptotic expansions of eigenvalues of nonselfadjoint singularly perturbed elliptic boundary value problems. Mat. Sb. (N.S.) 129(171) (1986), no. 3, 307-337. (Russian) MR 0837128 (87h:35262) 
[8] A. S. Demidov, Elliptic pseudodifferential boundary value problems with a small parameter multiplying the highest operator. Mat. Sb. (N.S.) 91(133) (1973), 421-444; English transl., Math. USSR-Sb. 20 (1973), no. 3, 439-463. MR0374726 (51:10922)

[9] _ Asymptotic behavior of the solution of a boundary value problem for elliptic pseudodifferential equations with a small parameter multiplying the highest operator. Trudy Moskov. Mat. Obšč. 32 (1975), 119-146. (Russian) MR0415691 (54:3771)

[10] G. I. Eskin, Asymptotic properties of the solutions of elliptic pseudodifferential equations with a small parameter. Dokl. Akad. Nauk SSSR 211 (1973), 547-550; English transl., Soviet Math. Dokl. 14 (1973), no. 4, 1080-1084. MR0425324 (54:13280)

[11] G. Grubb, Functional calculus of pseudo-differential boundary problems. Birkhäuser, Boston-BaselStuttgart, 1986. MR0885088 (88f:35002)

[12] M. S. Agranovich and M. I. Vishik, Elliptic problems with a parameter and parabolic problems of general type. Uspekhi Mat. Nauk 19 (1964), no. 3, 53-161. (Russian) MR0192188 (33:415)

[13] R. Denk, R. Mennicken, and L. R. Volevich, Boundary value problems for a class of elliptic operator pencils. Integ. Equations Operator Theory 8 (2000), 410-436. MR 1799649 (2001k:35128)

[14] _ On elliptic operator pencils with general boundary conditions. Integ. Equations Operator Theory 9 (2001), 25-40. MR1806842 (2002a:47020)

[15] B. A. Fuchs and V. I. Levin, Functions of a complex variable and applications. Gostekhizdat, Moskva, 1951; English transl., Pergamon Press, London, and Addison-Wesley, Reading, MA. 1961. MR0049291(14:150b) MR0132818(24:A2654)

[16] S. G. Gindikin and L. R. Volevich, Mixed problem for partial differential equations with quasihomogeneous principal part. Amer. Math. Soc., Providence, RI, 1996. MR1357662 (96j:35145)

[17] S. Agmon, A. Douglis, and L. Nirenberg, Estimates near the boundary for solutions of elliptic partial differential equations satisfying general boundary conditions. I. Comm. Pure Appl. Math. 12 (1959), 623-727. MR0125307 (23:A2610)

[18] L. R. Volevich and B. P. Paneyakh, Some spaces of generalized functions and embedding theorems. Uspekhi Mat. Nauk 20 (1965), no. 1, 3-74. (Russian) MR0174970 (30:5160)

[19] A. M. Il'in, Matching of Asymptotic Expansions of Solutions of Boundary Value Problems. Amer. Math. Soc., Providence, RI, 1992. MR.1182791 (93g:35016)

[20] L. R. Volevich, The Vishik-Lyusternik method in general elliptic boundary value problems with small parameter. Preprint, Keldysh Inst. Appl. Math., no. 26, 2002.

Keldysh Institute of Applied Mathematics, Russian Academy of Sciences, 4 Miusskaya Place, Moscow 125047, Russia

E-mail address: volevich@spp.keldysh.ru 